\title{
Persistent autism-relevant behavioral phenotype and social neuropeptide alterations in female mice offspring induced by maternal transfer of PBDE congeners in the commercial mixture DE-71
}

\author{
Elena V. Kozlova ${ }^{1,2}$ (1) Matthew C. Valdez ${ }^{1,2,8} \cdot$ Maximillian E. Denys $^{1} \cdot$ Anthony E. Bishay $^{1}$ (1) - Julia M. Krum ${ }^{1}$. \\ Kayhon M. Rabbani ${ }^{1}$. Valeria Carrillo ${ }^{1}$. Gwendolyn M. Gonzalez ${ }^{1}$. Gregory Lampel ${ }^{1}$. Jasmin D. Tran ${ }^{1}$. \\ Brigitte M. Vazquez ${ }^{1}$ - Laura M. Anchondo ${ }^{1}$. Syed A. Uddin ${ }^{1}$. Nicole M. Huffman ${ }^{1}$. Eduardo Monarrez ${ }^{1}$. \\ Duraan S. Olomi ${ }^{1}$. Bhuvaneswari D. Chinthirla ${ }^{1}$ - Richard E. Hartman ${ }^{4}$. Prasada Rao S. Kodavanti ${ }^{8}$. \\ Gladys Chompre ${ }^{5}$ Allison L. Phillips ${ }^{3} \cdot$ Heather M. Stapleton $^{3} \cdot$ Bernhard Henkelmann $^{6} \cdot$ Karl-Werner Schramm $^{6,7}$ (D) \\ Margarita C. Curras-Collazo ${ }^{1}[$
}

Received: 11 June 2021 / Accepted: 16 September 2021 / Published online: 23 October 2021

(c) The Author(s) 2021

\begin{abstract}
Polybrominated diphenyl ethers (PBDEs) are ubiquitous persistent organic pollutants (POPs) that are known neuroendocrine disrupting chemicals with adverse neurodevelopmental effects. PBDEs may act as risk factors for autism spectrum disorders (ASD), characterized by abnormal psychosocial functioning, although direct evidence is currently lacking. Using a translational exposure model, we tested the hypothesis that maternal transfer of a commercial mixture of PBDEs, DE-71, produces ASD-relevant behavioral and neurochemical deficits in female offspring. C57B16/N mouse dams (F0) were exposed to DE-71 via oral administration of $0(\mathrm{VEH} / \mathrm{CON}), 0.1$ (L-DE-71) or 0.4 (H-DE-71) $\mathrm{mg} / \mathrm{kg}$ bw/d from 3 wk prior to gestation through end of lactation. Mass spectrometry analysis indicated in utero and lactational transfer of PBDEs (in ppb) to F1 female offspring brain tissue at postnatal day (PND) 15 which was reduced by PND 110. Neurobehavioral testing of social novelty preference (SNP) and social recognition memory (SRM) revealed that adult L-DE-71 F1 offspring display deficient shortand long-term SRM, in the absence of reduced sociability, and increased repetitive behavior. These effects were concomitant with reduced olfactory discrimination of social odors. Additionally, L-DE-71 exposure also altered short-term novel object recognition memory but not anxiety or depressive-like behavior. Moreover, F1 L-DE-71 displayed downregulated mRNA transcripts for oxytocin $(O x t)$ in the bed nucleus of the stria terminalis (BNST) and supraoptic nucleus, and vasopressin $(A v p)$ in the BNST and upregulated Avplar in BNST, and Oxtr in the paraventricular nucleus. Our work demonstrates that developmental PBDE exposure produces ASD-relevant neurochemical, olfactory processing and behavioral phenotypes that may result from early neurodevelopmental reprogramming within central social and memory networks.
\end{abstract}

Keywords Endocrine-disrupting chemicals · Developmental exposure $\cdot$ Oxytocin $\cdot$ Flame retardants $\cdot$ Polybrominated diphenyl ethers $\cdot$ Vasopressin

This report is dedicated to Dr. Elizabeth R. Gillard, who set us on the path to study the neurotoxicity of endocrine disrupting chemicals and who embodied an intense passion for discovery. Moreover, she cultivated an inclusive culture of mentorship and training, tirelessly, followed scientific leads and adhered to a herculean work ethic that has promoted the highest standards for excellence in the lab. We immortalize her memory here.

Margarita C. Curras-Collazo

mcur@ucr.edu

Extended author information available on the last page of the article

Abbreviations
AMG
ASD
AVP
Avp1ar
BDE
BFR
BNST
BPA
DE-71

Amygdala

Autism spectrum disorders

Arginine-8-vasopressin

Arginine-8-vasopressin receptor

Brominated diphenyl ether

Brominated flame retardants

Bed nucleus of the stria terminalis

Bisphenol A

Pentabromodiphenyl oxide 


$\begin{array}{ll}\text { ECNI-MS } & \text { Electron capture negative ion mass } \\ \text { spectrometry } \\ \text { EDC } & \text { Endocrine disrupting chemical } \\ \text { EPM } & \text { Elevated plus maze } \\ \text { FST } & \text { Forced swim test } \\ \text { GOI } & \text { Gene of interest } \\ \text { LS } & \text { Lateral septum } \\ \text { MIA } & \text { Maternal immune activation } \\ \text { NDD } & \text { Neurodevelopmental disorders } \\ \text { NORT } & \text { Novel object recognition test } \\ \text { OFT } & \text { Open field test } \\ \text { OPT } & \text { Olfactory preference test } \\ \text { OXT } & \text { Oxytocin } \\ \text { Oxtr } & \text { Oxytocin receptor } \\ \text { PACAP (Adcyap1) } & \text { Pituitary adenylate cyclase-activat- } \\ & \text { ing polypeptide } \\ \text { PAC1r (Adcyap1r) } & \text { Pituitary adenylate cyclase-activat- } \\ & \text { ing polypeptide 1 receptor } \\ \text { PBDE } & \text { Polybrominated diphenyl ether } \\ \text { PND } & \text { Postnatal day } \\ \text { PVN } & \text { Paraventricular nucleus of the } \\ & \text { hypothalamus } \\ \text { ROI } & \text { Region of interest } \\ \text { SRMT } & \text { Social recognition memory test } \\ \text { SNP } & \text { Single-nucleotide polymorphisms } \\ \text { SOC } & \text { Sociability } \\ \text { SON } & \text { Supraoptic nucleus of the } \\ & \text { hypothalamus } \\ \text { SRM } & \text { Social recognition memory } \\ \text { SVZ } & \text { Subventricular zone } \\ \text { VMN } & \text { Ventromedial hypothalamus } \\ & \end{array}$

\section{Introduction}

Autism spectrum disorder (ASD) is a group of neurodevelopmental conditions defined clinically by deficits in social reciprocity and communication, and restricted interest and repetitive behaviors (American Psychiatric Association 2013). Hallmarks of ASD, as classified by the NIH Research Domain Criteria (RDoC) (Social Processes: Workshop Proceedings, 2012) include disturbances in the social cognition (SC) domain such as facial recognition ability, empathy and evaluation of emotions of others (Weigelt et al. 2012); (Ewbank et al. 2017). The prevalence of ASD has increased dramatically over the past 3 decades. In the United States, the Centers for Disease Control (CDC) estimates that ASD affects 1 in 54 neurotypical children (Maenner et al. 2020), while the worldwide prevalence is estimated to be $1-2 \%$ (Kim et al. 2011). While genetic heritability is an important factor in ASD etiology, the incremental incidence of autism over the last several decades, raises the possibility that environmental factors, such as xenobiotic chemicals, may contribute alongside genetic predisposition and influence ASD risk (Grandjean and Landrigan 2014); (Pelch et al. 2019). Although the incidence of autism is four times greater in boys, girls and women with autism are often undiagnosed, misdiagnosed or receive a diagnosis of autism at later age (Rynkiewicz et al. 2019) suggesting underestimation in females. According to the female protective model, females may also benefit from a higher threshold of genetic liability to manifest ASD phenotype (Werling and Geschwind 2013); (Zhang et al. 2020). Nevertheless, female ASD cases may display susceptibility to xenobiotic chemicals (Terasaki et al. 2016) that can potentially enhance the risk of neurodevelopmental disorders (NDDs). Indeed, we have found that female mice offspring exposed to PBDEs during prenatal and postnatal development exhibit endocrine and metabolic disruption, indicating that females may provide a susceptible substrate for studying xenobiotic effects on neurodevelopment (Kozlova et al. 2020).

Polybrominated diphenyl ethers (PBDEs) are a class of brominated flame retardants added to a wide range of products including consumer building material, electronics, textiles, plastics and foams including infant products (Ionas et al. 2016) since the 1970s (Stapleton et al. 2005). Three commercial formulations of PBDEs were prevalent in commerce, including penta-BDE, octa-BDE and deca-BDE. Two commercial PBDE mixtures, penta- and octa-BDEs, were banned in Europe in 2003 and all PBDEs were voluntarily phased out in the US by 2013 , leading to a slow, but measurable, decrease in environmental levels as well as in human sera and breastmilk concentrations of some PBDE congeners (Drage et al. 2019); (Guo et al. 2016). Notwithstanding a commitment to a voluntary phase out of decaBDE by 2013, PBDE contamination is predicted to remain an ongoing problem through the next several decades due to their long half-lives, persistence in e-waste (Ohajinwa et al. 2019), recycling into consumer products and inadvertent reappearance into environment (Abbasi et al. 2019). In an unprecedented action, the U.S. EPA formally banned the production, import and distribution of deca-BDE in February 2021. Nevertheless, PBDEs are still being detected in various tissue samples worldwide, including human breastmilk (Terry et al. 2017); (Hurley et al. 2017); (Lyche et al. 2015); (Chen et al. 2014); (Darrow et al. 2017).

Compared to adults, infants and toddlers are at greater risk of the adverse health effects resulting from PBDE exposure since they disproportionately accumulate 3- to 9-fold greater body burdens (Costa et al. 2014). Circulating levels of PBDEs in US children are 10- to 1000-fold higher than similar age populations in Mexico and Europe (Rose et al. 2010). Elevated exposures in infants are due to the maternal transfer of PBDEs via cord blood and breastmilk (Toms et al. 2008). After weaning in early childhood, an additional route of exposure is dust ingestion and inhalation associated 
with children's mouthing and crawling behaviors (Stapleton et al. 2008); (Johnson-Restrepo and Kannan 2009). Therefore, high PBDE exposure poses significant health risks during critical periods of development.

Major health effects associated with PBDE exposures are endocrine disruption, reproductive and developmental toxicity and neurotoxicity (Costa and Giordano 2007); (Darnerud 2008); (Kodavanti and Curras-Collazo 2010); (Kodavanti et al. 2010); (Dingemans et al. 2011). However, epidemiological studies examining the association between PBDE exposure and ASD show inconsistent findings. PBDE exposure (e.g., PBDE congeners BDE-153 and -47) during both pre- and post-natal development has been linked to adverse neurological outcomes such as impairments in executive function, poor attention and behavioral regulation, reduced social scores, and lower IQ. Early-life exposure to PBDEs (BDE-47, -99 and/or -100) has been associated with externalizing behaviors such as hyperactivity and impulsivity (Roze et al. 2009); (Ding et al. 2015); (Herbstman et al. 2010); (Hoffman et al. 2012); (Vuong et al. 2018). With regard to the association of PBDEs with social behavior deficits and ASD, preschool-aged children with greater $\Sigma \mathrm{PBDE}$ exposures were rated as less assertive by their teachers (Lipscomb et al. 2017) or showed greater anxious behavior (Adgent et al. 2014). In the HOME prospective cohort study, serum levels of PBDEs in mothers were associated with greater (BDE-28) or fewer (BDE-85) autistic behaviors in their children (Braun et al. 2014). Similarly, significantly higher risk of poor social competence symptoms was shown as a consequence of postnatal BDE47 exposure (Gascon et al. 2011). Although the possibility that environmental toxicants serve as risk factors for social neurodevelopmental disorders (NDDs) has not been established (Messer 2010), PBDEs may have deleterious effects on children's social development relevant to ASD (Ding et al. 2015); (Messer 2010; Gascon et al. 2011; Braun et al. 2014); (Gibson et al. 2018). Studies in experimental animals demonstrate that certain PBDE congeners produce adverse effects on behavior, learning, and memory in exposed offspring (Costa and Giordano 2007); (Kodavanti and CurrasCollazo 2010); (Pinson et al. 2016), but information about the negative impact of PBDEs on psycho-social behavior is limited (Woods et al. 2012); (Kim et al. 2015). We hypothesized that developmental PBDE exposure produces ASDrelevant social behavioral and neurochemical phenotypes in a mouse toxicant model.

Social recognition, or the ability to distinguish between familiar and novel conspecifics, is a fundamental process across species required for forming long-term attachments, hierarchies, and other complex social strategies that enhances survival (Brennan and Kendrick 2006). Disturbances in this capacity are present in individuals with ASD who have difficulties identifying faces of novel conspecifics from those previously encountered (Weigelt et al. 2012); (Ewbank et al. 2017). Rodents, because of their highly social nature, are used as proxies for studying autism-relevant social competence (Young et al. 2002). Mouse social behavior paradigms rely on the natural propensity of mice for investigation of social novelty compared to previously encountered conspecifics when given the choice (Moy et al. 2004). This preference for social novelty has been shown to be absent in monogenetic, idiopathic and environmental models of ASD (Silverman et al. 2010); (Sgritta et al. 2019); (Buffington et al. 2016)). In the current study, we used a toxicant exposure mouse model to characterize social recognition ability, repetitive behaviors and concomitant autism comorbidities such as anxiety, memory impairment and altered olfactory processing.

While the behavioral deficits in typical ASD rodent models are well established, the underlying neural mechanisms are not well understood. The neuropeptides oxytocin and vasopressin are considered major neurotransmitters implicated in social information processing and social cognition that are disrupted in ASD patients (Landgraf and Neumann 2004). Rodent studies have shown that these neuropeptidergic systems are involved in several social cognition domains such as social memory, social/emotional recognition and social reward (Ferguson et al. 2001); (Raam et al. 2017); (Bielsky et al. 2004); (Ferretti et al. 2019). Work by us and our collaborators has provided evidence that PBDEs (and the structural analogues, polychlorinated biphenyls (PCBs)) disrupt the magnocellular neuroendocrine system responsible for vasopressin production involved in osmoregulation, cardiovascular function and social behavior (Kodavanti and Curras-Collazo 2010); (Coburn et al. 2005); (Coburn et al. 2007); (Currás-Collazo 2011); (Coburn et al. 2015); (MucioRamírez et al. 2017); (Alvarez-Gonzalez et al. 2020). We have shown that exposure to DE-71 during in utero and lactation via maternal transfer can nearly abolish vasopressin immunoreactivity in the activated supraoptic (SON) and paraventricular nuclei (PVN) of the hypothalamus (Coburn et al. 2007); (Mucio-Ramírez et al. 2017). Therefore, we also tested the hypothesis that PBDEs disrupt gene expression of prosocial neuropeptides such as vasopressin, oxytocin, PACAP and their receptors in regions of the social brain network, which may underlie deficient social behavior (Bicks et al. 2020); (Tanimizu et al. 2017); (Ferguson et al. 2000).

To lend insight to whether early-life exposure to PBDEs can produce ASD-relevant phenotypes, we exposed mouse dams to a commercial mixture of PBDEs, DE-71, at low doses to mimic chronic, low-level exposure to BDE congeners and doses encountered by infants and toddlers. We demonstrate that perinatal exposure to DE-71 produces dosedependent deficits in social recognition memory and general memory, altered olfactory function and altered neuromolecular phenotypes in brain regions that coordinate complex 
social behaviors. To the best of our knowledge, this study is the first to show a comprehensive profile of autistic-relevant behavior, comorbidities and brain transcriptional changes in female offspring impacted by maternal transfer of PBDEs. Concomitant characterization of ASD-relevant behavioral and neurochemical phenotypes exhibited by offspring developmentally exposed to and reprogrammed by DE-71, provides an integrative framework for exploring environmental risk factors that may contribute to the increasing incidence of ASD. A portion of our findings has been published in preliminary form (Kozlova et al., 2019).

\section{Materials and methods}

\section{Animal housing and care}

C57B1/6 N mice were generated using breeders obtained from Charles River Labs (West Sacramento, CA). Mice were housed 2-4 per cage in standard polycarbonate plastic cages with corn-cob bedding in a specific pathogen-free vivarium and kept on a 12:12h light:dark cycle in a controlled temperature $\left(21.1-22.8{ }^{\circ} \mathrm{C}\right)$ and humidity $(20-70 \%)$ environment. Mice were provided rodent chow (Laboratory Rodent Diet 5001; LabDiet, USA) and water ad libitum. Care and treatment of animals was performed in compliance with NIH guidelines and approved by the University of California, Riverside Institutional Animal Care and Use Committee (AUP\# 20170026 and 20200018).

\section{DE-71 exposure and experimental design}

DE-71 (technical pentabromodiphenyl oxide; Lot no. 1550OI18A), was obtained from Great Lakes Chemical Corporation (West Lafayette, IN). Ninety-seven percent of this mixture contains the following congener composition (in \%): BDE-17 (0.11), -28 (0.24), -47 (33.3), -85 (2.54), -99 (45.3), - 100 (8.24), - 138 (0.45), - 139 (0.92), - 153 (3.57), -154 (3.19) as described (Kodavanti et al. 2010). DE-71 dosing solutions were prepared in corn oil vehicle (VEH/CON) to yield two doses: $0.1 \mathrm{mg} / \mathrm{kg} / \mathrm{d}$ (L-DE-71) and $0.4 \mathrm{mg} / \mathrm{kg} / \mathrm{d}$ (H-DE-71) using $2 \mathrm{~mL}$ of stock solution $/ \mathrm{kg}$ body weight. The DE-71 doses were selected to contain the same molar concentrations of BDE-47 used in other mouse studies (Woods et al. 2012); (Wang et al. 2018). BDE-47 has been the focus of many PBDE studies, in part, because it is the primary congener found in human breast milk (Guo et al. 2016); (Darnerud et al. 2015).

Offspring were exposed to DE-71 via maternal transfer using a 10-week dosing regimen (Fig. 1a) as described previously (Kozlova et al. 2020). Mice were randomly assigned to one of the three exposure groups: corn oil vehicle control (VEH/CON), L-DE-71 or H-DE-71. This exposure paradigm was chosen to model chronic, low-level exposure to the mother and transfer of PBDEs to infant during gestation (1st, 2nd and 3rd trimester) and lactation as shown in humans (Toms et al. 2008); (Schecter et al. 2007); (Chao et al. 2011); (Zhao et al. 2013). After 3 weeks of pre-dosing, virgin females were paired with an untreated male using harem-style breeding. The presence of a vaginal plug was designated as gestational day (GD) 0 . Females that failed to conceive within $10 \mathrm{~d}$ were removed from the study. The litters were not culled to avoid potential artificial equalization of variance that masks the developmental and reproductive effects of toxicants as justified previously (Suvorov and Vandenberg 2016). F1 offspring were weaned at PND21 and housed in same-sex cages (2-4/cage). Dams (F0) and their adult female offspring (F1) were subjected to behavioral testing and later sacrificed by exsanguination via cardiac puncture under terminal isoflurane anesthesia (5\%) followed by cervical dislocation.

To reduce cross-over effects, behavioral tests were distributed across three different cohorts. Mice were run through a battery of behavioral tests in the following order for Cohort 1 (mean age): Suok (PND 46); social novelty preference test (SNP; PND 71); 3 chamber social novelty (PND 87); elevated plus maze (EPM; PND 72). The brains of Cohort 1 were collected at sacrifice on PND 108 and used in RTqPCR. The following tests were performed on Cohort 2: Marble burying (MB; PND 81); olfactory habituation/dishabituation (OHT; PND 79); olfactory preference (OPT; PND 102); forced swim test (FST; PND 74). Cohort 3 was subjected to social recognition memory (SRMT; PND 30); juvenile open field (OFT; PND 31); juvenile MB (PND 35); novel object recognition (NORT; PND 111) tests. F1 and F0 were tested similarly, except that F0 did not get tested on the SRMT. Analytical characterization by mass spectrometry was performed on brains from Cohort 1 (PND 110) and a subset of Cohort 3 (PND 15). Enzyme-linked immunosorbent assays (ELISA) were performed on plasma from Cohorts 1-3. Whenever possible, the dam or litter was used as the statistical unit of analysis for F1 i.e. values of offspring in each litter were averaged to represent one sample. This allowed us to control for large differences between litters if they existed (Jiménez and Zylka 2021). Based on our previous findings we estimate 5.8, 5.8, 7.2 pups/litter for VEH/CON, L-DE-71 and H-DE-71, respectively, with no differences in the secondary sex ratio (Kozlova et al. 2020). Therefore, there were on average three females per litter per group. A total of 45 litters, distributed equally across experimental groups, were needed to cover the range of experiments conducted in these studies. The number of litters per Cohort is as follows: Cohort 1 (17), Cohort 2 (18), Cohort 3 (10). In addition, results were replicated in a minimum of three independent experiments. 
Fig. 1 Maternal dosing paradigm for DE-71 produces BDE congener penetration in female F1 offspring brain. a Dosing and testing paradigm used for perinatal and adult exposure to DE-71. Direct exposure to DE-71 in adult dams (F0우; solid shading), began 3-4 weeks pre-conception and continued until pup weaning at PND 21. Indirect exposure in female offspring (F1\%; hatched shading) occurred perinatally (GD 0 to PND 21). b The ng/g wet wt (ww) sum concentrations of the 14 PBDE congeners ( $\sum_{14}$ PBDE) detected at PND 15. c The ng/g wet wt sum concentrations of the $1 \mathrm{PBDE}$ congener, BDE, 153, detected at PND 110. d BDE composition (\% total) in DE-71 and in brains of exposed female offspring obtained at PND 15 and PND 110. The 7 congeners that comprise $<1 \%$ of DE-71 were displayed as $1 \%$. e,f Absolute congener concentrations at PND 15 and PND 110 for L- and H-DE-71. All values for VEH/ CON were $<$ MDL (not shown). $* P<.05, * * P<.01$ compared to $\mathrm{VEH} / \mathrm{CON} ;{ }^{\wedge} P<.05$ compared to L-DE-71. $n=3-4$ /group. $G D$ gestational day, $P N D$ postnatal day

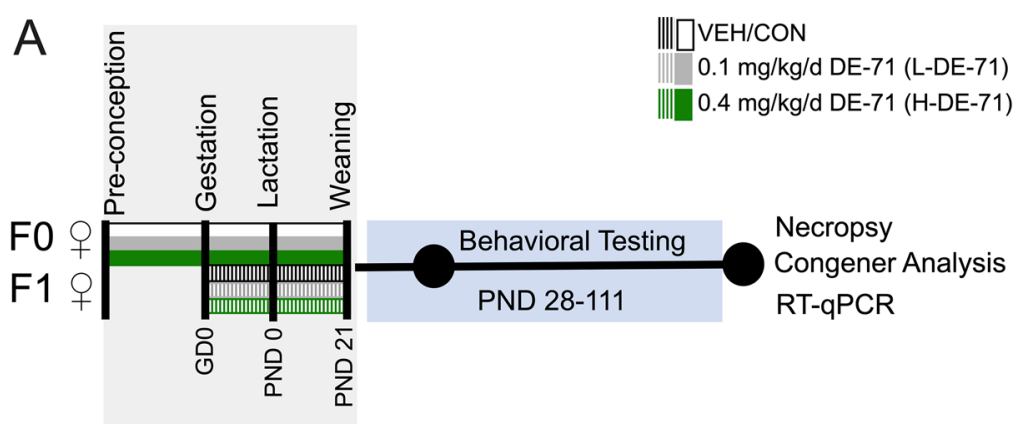

B
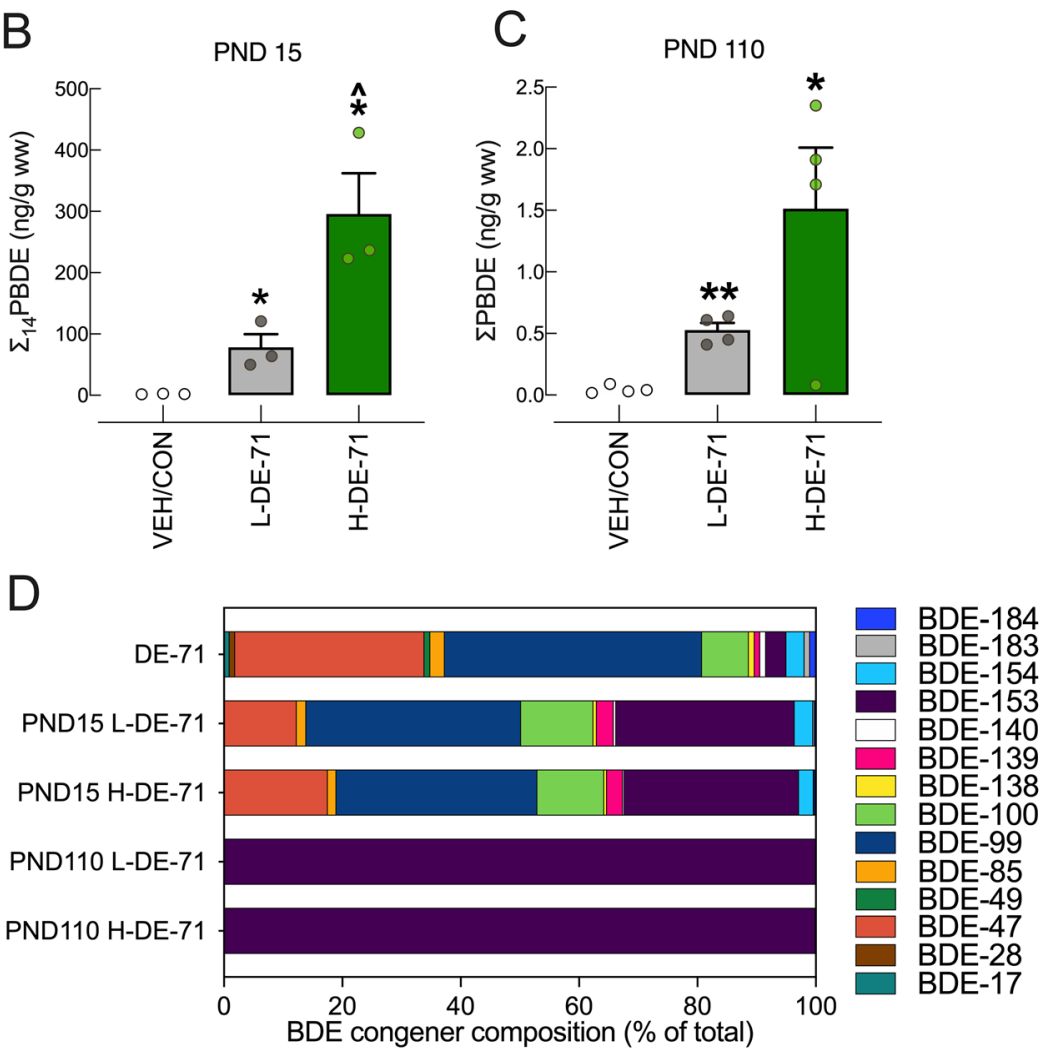

E

PND 15
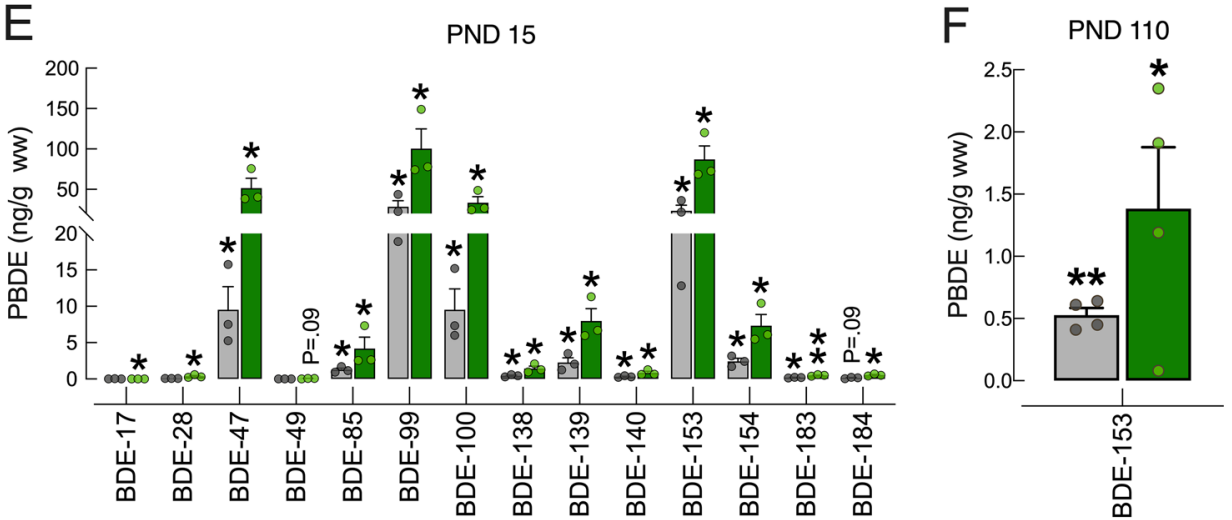


\section{Nest scoring}

To test for possible effects of DE-71 on maternal parameters, nests of single-housed dams built from pressed cotton squares $(5 \times 5 \mathrm{~cm}$; Nestlets) were evaluated at PND 0-1 using a modified scoring system (Hess et al. 2008). Manual scores were assigned by several experimenters according to the height and closure of the walls surrounding the nest cavity. Scores were assigned according to whether the nest contained a center (1) plus a 50\% border, (2) $75 \%$ border (3) or $100 \%$ border (4). A score of 5 was given if the nest resembled a dome (Supplementary Fig. 1). Nest scores were boosted by 0.5 if the nest was elevated. For interrater reliability, the Bland-Altman method was used to calculate bias as the mean of the differences ( 0 representing two judges were not producing different results) and precision as $95 \%$ limits of agreement (standard deviation of mean bias \pm 1.96 ) (Supplementary Fig. 1).

\section{Congener analysis in adult offspring brain}

PBDE concentrations were measured in PND110 whole brain homogenate extracts by the Stapleton laboratory at Duke University using gas chromatography coupled with electron capture negative ion mass spectrometry (GC/ECNIMS; Agilent 5975 N MS) as described previously (Kozlova et al. 2020). Briefly, approximately $0.2-0.5 \mathrm{~g}$ of tissue was first ground with clean sodium sulfate, spiked with two isotopically labeled standards (F-BDE-69 and 13C BDE-209) and then extracted using 50:50 DCM:hexane. Extracts were concentrated, measured for lipid content using a gravimetric analysis, and then purified using acidified silica before analysis for 26 different PBDE congeners ranging from BDE-30 to BDE 209. Laboratory processing blanks (clean sodium sulfate only) were analyzed alongside samples to monitor background contamination. Recoveries of F-BDE-69, and 13C BDE 209, averaged $91( \pm 6.9 \%)$ and $106( \pm 19.9 \%)$, respectively, in all samples. All samples were blank-corrected on a congener-specific basis using the average concentrations measured in the laboratory processing blanks. Method detection limits (MDLs) were estimated using either a signal to noise ratio of 10 , or, if analytes were detected in laboratory blanks, by calculating three times the standard deviation of the laboratory blanks. MDLs differed by congener and ranged from 0.8 (BDE-47) to $6.6 \mathrm{ng} / \mathrm{g}$ (BDE-206).

\section{PBDE congener analysis in postnatal offspring brain}

Due to force majeure, i.e. SARS-CoV-2 pandemic, we were unable to carry out planned analytical characterization of PND 15 tissues in collaboration with the Stapleton lab, therefore, the mass spectrometry (MS) system in the Schramm lab was used. The performance of both methods was comparable, especially with regard to the limit of quantification. Using high-resolution gas chromatography-high-resolution mass spectrometry (HRGC/HRMS), PBDE concentrations were measured in PND15 whole brain homogenates (0.1-0.2 g) as described (Li et al. 2020). PBDE analytes included 37 PBDE congeners (BDE-7, 10, 15, 17, 28, 30, $47,49,66,71,77,85,99,100,119,126,138,139,140,153$, 154, 156, 176, 180, 183, 184, 191, 196, 197, 201, 203, 204, 205, 206, 207, 208, 209). Samples were ground and homogenized to a fine powder under liquid nitrogen. Each sample (100-200 mg) was mixed with CHEM TUBE-Hydromatrix (Agilent Technologies) and spiked with ${ }^{13} \mathrm{C}$-labelled PBDE standard mix (BFR-LCS, Wellington Laboratories). For pressurized liquid extraction (Dionex ASE 200) n-hexane/ acetone $(3: 1, \mathrm{v} / \mathrm{v})$ was used at $120^{\circ} \mathrm{C}$ and $12 \mathrm{MPa}$. The volume of the extract was reduced to $\sim 5 \mathrm{~mL}$ using a vacuum rotary evaporator. Samples were purified using an automated system (DEXTech, LCTech, Germany), where the sample was passed and fractionated over an acidic silica, alumina and carbon column. Concentrated extracts were spiked with the recovery standard (BFR-SCS, Wellington Laboratories) and analyzed by HRGC/HRMS (Agilent 6890/Thermo MAT95 XL) using electron impact ionization (EI), in the selected ion monitoring mode. The instrumental parameters are listed in Supplementary Table 1. Average recovery for ${ }^{13} \mathrm{C}$-labelled PBDE standards ranged between 40 and $120 \%$. All samples were blank-corrected on a congener-specific basis using the average of three procedural blank samples. Analytes with concentrations after blank correction that were lower than three times the standard deviation of the blank values or that were not detected before blank correction were considered as not detectable (n.d.). The limit of quantification (LOQ) of the instrumental methodology was considered as a signal/noise ratio of 9:1 (Supplementary Table 2). Congener concentrations that were below the detection limit were assigned a randomly generated value of LOQ/2. The accuracy of our method was confirmed by successful participation in interlaboratory comparison studies.

\section{Comparison of MS methods}

The GC/ECNI-MS method used the ECNI ionization mode to improve sensitivity and provides equal sensitivity to HRGC/HRMS that uses electron impact.

\section{Neurobehavioral testing paradigms}

At least 30 min prior to testing, mice were moved to a designated behavior room. Ethanol (70\%) was used to remove debris and odors from apparati between individual mouse trials. Unless stated otherwise, mouse behavior was scored using automated video-tracking software (Ethovision XT 15, Noldus) or manual scoring software (BORIS (Friard and 
Gamba 2016) or JWatcher), performed blind to treatment by trained observers. Mice were tested between $10 \mathrm{am}$ and $4 \mathrm{pm}$ during the light phase under bright light conditions, unless otherwise stated.

\section{Social novelty preference}

Social novelty preference (SNP) was conducted and analyzed according to methods adopted from published protocols (Moy et al. 2004). Briefly, mice were habituated for $30 \mathrm{~min}$ to a polycarbonate cage identical to their home cage ( $26 \mathrm{~cm}$ length $\times 16$ width $\mathrm{cm} \times 12 \mathrm{~cm}$ height), followed by $30 \mathrm{~min}$ to two wire interaction corrals $(11 \mathrm{~cm}$ height $\times 10 \mathrm{~cm}$ diameter) placed on each side of the cage. During a 5-min training trial, a stimulus mouse was placed into one corral while the empty corral was removed. After a 30 min retention period, social recognition was assessed in the following 5 min test, during which the test mouse explored the same stimulus mouse (now familiar mouse) versus a novel stimulus mouse. Prior to testing days, sex- and age-matched conspecific stimulus mice were trained to stay in corrals for $15 \mathrm{~min}$ for 3 times per day for 7-14 d. Stimulus mice were single-housed to preserve their unique scent. Investigation by test mouse was measured as time spent sniffing (snout within $2 \mathrm{~cm}$ of stimulus). Test robustness was measured using an Investigation Index calculated as the ratio of time spent investigating the novel mouse to total investigation time during the training trial (Supplementary Fig. 2). Social recognition is represented as time spent investigating novel stimulus as percent of total investigation time during test trial. To evaluate between group differences, a Recognition Index was calculated as the time spent investigating Novel minus Familiar/total investigation time in test period.

\section{Three-chamber sociability task}

Sociability was assessed as described (Yang et al. 2011). In brief, during the first habituation phase, test mice were habituated for $10 \mathrm{~min}$ to the center chamber of a Plexiglass three-chambered apparatus $(22 \times 40 \times 23 \mathrm{~cm})$. Next, the retractable doors partitioning the chambers were opened to permit exploration of all three chambers (second habituation phase). Sociability was tested in the following $10 \mathrm{~min}$ session, during which the test mouse was permitted to explore an empty $9 \times 27 \mathrm{~cm}$ corral (novel object) versus a different corral occupied by a stimulus mouse (novel social object). Inherent side preference during the second habituation phase was evaluated as right chamber time minus left chamber time/right chamber time + left chamber time $\times 100$. Mean values for test mice meeting the inclusion criterion $(0 \pm 15 \%)$ are shown in Supplementary Fig. 2. Sociability was analyzed during the subsequent testing phase both as time spent in chamber and time spent sniffing within $2 \mathrm{~cm}$ of stimulus.

\section{Marble burying and nestlet shredding tests}

The marble burying (MB) and nestlet shredding tests were utilized for analysis of elicited repetitive behavior in rodents that is considered analogous to that observed in autistic individuals (Silverman et al. 2010). During the marble burying test, the test mouse was placed in the corner of a polycarbonate cage $(19 \times 29 \times 13 \mathrm{~cm})$ containing $5 \mathrm{~cm}$ of bedding (Angoa-Pérez et al. 2013) and allowed to interact for $30 \mathrm{~min}$ with an array of equidistant marbles $(8 \times 4$ for adults or $5 \times 4$ for juvenile). A minimum $2 / 3$ of the marble was defined as being buried in the 32 -marble array and $1 / 2$ buried in the 20 -marble array. Images of the cage were scored by $2-3$ experimenters who were blind to treatment and a mean score obtained. For interrater reliability on marble burying the Bland-Altman method was used to calculate bias as the mean of the differences ( 0 representing that two judges were not producing different results) and precision as $95 \%$ limits of agreement (standard deviation of mean bias \pm 1.96 ) (Supplementary Fig. 3). After a 5 min rest period, the test mouse was placed into another cage of the same size with $0.5 \mathrm{~cm}$ of bedding containing a pre-weighted square of cotton fiber (Nestlet). After $30 \mathrm{~min}$, the remaining Nestlet was weighed and percent shredding calculated.

\section{Social recognition memory test}

A two-trial social recognition memory test (SRMT) was performed as previously described (Tanimizu et al. 2017) to assess long-term social recognition memory. Test mice (PND 28-40) were exposed to a juvenile sex-matched conspecific stimulus mouse (PND 15-32) during two 3 min trials following an intertrial delay of $24 \mathrm{~h}$. For each experiment, test mice were individually placed into polycarbonate cages $(26 \times 16 \times 12 \mathrm{~cm})$ and allowed to habituate for $1 \mathrm{~h}$ under dim conditions. A juvenile sex-matched conspecific was then placed into the cage, and the mice were allowed to interact for 3 min during trial 1 (Day 1). In trial 2, performed $24 \mathrm{~h}$ later on Day 2, the same test mouse was exposed to the same stimulus mouse (familiar from Day 1). Each stimulus was not used more than 4 times per day. The tests were digitally recorded and scored for social investigation behavior. To evaluate the differences in the ability of mice to form a long-term social memory a Recognition Index (RI) was calculated as the ratio of the duration of investigation of stimulus mouse on Day 2 over Day 1. We used a one-sample $t$ test to determine if the mean RI of each group was statistically different from the previously reported mean RI of 0.65 (Kogan et al. 2000); (Tanimizu et al. 2017). In a second set of experiments, test 
mice were exposed to a novel stimulus mouse on Day 2 . In this context, we used a one-sample $t$ test to determine if the sample mean RI was statistically different from 1 , representing similar preference for mouse presented on Day 1 and new novel stimulus mouse presented on Day 2.

\section{Novel object recognition test}

The novel object recognition test (NORT) was used to assess non-social recognition memory. We adapted an established protocol to a two-day protocol that tested the same mouse using short- and long-term retention times. On Day 1, the test mouse was habituated to an empty square Plexiglas open field arena $(39 \times 39 \times 38 \mathrm{~cm})$ for $15 \mathrm{~min}$ as described (Murai et al. 2007), followed by a 20 min rest in its home cage. During the acquisition phase, the test mouse was placed in the open field containing two identical objects (F vs $F^{\prime}$ ) and allowed to freely explore the environment and objects. During the short-term memory (30 min retention) testing session, the test mouse was again placed in the apparatus and allowed to explore a familiar and novel object (F vs N). After a 24 h retention time (Day 2), long-term memory was assessed by placing mice into the open field containing both the familiar and a new novel object (F vs $\mathrm{N}^{\prime}$ ). All test/train sessions lasted $5 \mathrm{~min}$. Preference for the novel object was expressed as the ratio of time exploring the novel stimulus relative to the total exploration time. To evaluate the differences in ability to form NOR memory, a Discrimination Index was calculated as the difference in exploration time between novel and familiar objects relative to total exploration time, where 0 indicates equal preference. Test objects were first validated for intrinsic preference in untreated mice. After analysis of the data using Ethovision, we applied the following exclusion criteria: a) total distance travelled one or more standard deviation(s) lower than the group mean for any trial, or b) less than 6 visits to the familiar or novel target zones.

\section{Innate olfactory preference test}

To test the ability of mice to detect attractive or aversive odorants, the innate Olfactory Preference Test (OPT) was performed and analyzed as described (Kobayakawa et al. 2007). Mice were habituated to the experimental conditions. First, they were placed individually into an empty test cage $(19 \times 29 \times 13 \mathrm{~cm})$ and sequentially transferred to three other cages every $15 \mathrm{~min}$. After the final habituation, mice were transferred into the test cage containing a filter paper $(2 \times 2 \mathrm{~cm})$ infiltrated with either $500 \mathrm{uL}$ of a fresh solution of test odorants: $10 \%$ peanut butter, $1 \%$ vanilla, $1 \%$ butyric acid, or deionized water. The four test odorants were presented to the test mouse in a randomized order. Time spent sniffing the filter paper during the 3-min odorant trials was video-recorded and later measured.

\section{Olfactory habituation test}

The ability of mice to detect and differentiate social and nonsocial odorants was examined using the olfactory habituation/dishabituation test (OHT) (Silverman et al. 2010). OHT involves presenting a test animal with various non-social and social odorants. Mice were acclimated for $45 \mathrm{~min}$ to an empty mouse cage $(26 \times 16 \times 12 \mathrm{~cm})$ and lid with a cotton-tipped applicator inserted through the water bottle hole to reduce the novelty of the applicator during test sessions. Non-social odors were prepared from extracts immediately before testing. They included: (1) deionized water; (2) almond (1:100; McCormick); (3) banana (1:100; McCormick). Two social odors were obtained the morning of test day by swiping applicator across the bottom of two different stimulus mouse cages containing soiled bedding from sex-matched conspecifics. Cages housed 3-4 mice and bedding was at least $3 \mathrm{~d}$ old. Stimuli were presented in 2-min triplicate trials in the following order: water, almond, banana, social odor 1, social odor 2. Time spent sniffing the applicator was recorded with a stopwatch. Parameters measured were habituation, defined as a decrement in olfactory investigation of the same odor after repeated presentations and dishabituation, defined as a reinstatement of olfactory investigation upon presentation of a new odorant.

\section{Suok}

Suok is an elevated platform behavioral paradigm used to analyze anxiety, anxiety-induced motor impairments and motor-vestibular anomalies in mice. The apparatus consists of a smooth aluminum beam ( $2 \mathrm{~m}$ long, $3 \mathrm{~cm}$ diameter) elevated to $20 \mathrm{~cm}$ and fixed to two clear acrylic walls as described (Kalueff et al. 2008). Bilateral to a central segment $(38 \mathrm{~cm})$ of the aluminum rod are $10 \mathrm{~cm}$ segments labeled by line markings. After acclimation to the dimly lit testing room, mice were placed in the center of the beam and several behaviors were scored over a 5 min trial: (1) horizontal and locomotor (normalized) activity, assessed by number of segments traveled, (2) sensorimotor coordination, measured by the number of hind leg slips and falls from the rod, (3) exploratory behavior such as side looks and head dips, (4) anxiogenic behaviors such as increased latency to leave the central zone and unprotected stretch attend postures (SAP), in which the mouse stretches forward and retracts without moving its feet (considered a non-social form of ambivalence), (5) vegetative responses (combined number of urinations and defecation boli), and (6) autogrooming behaviors . 
Hyperactivity, loss of sensorimotor coordination, increased anxiety and displacement behavior are represented by elevated values for \#1, 2, 4 and \#5, and 6, respectively. Locomotor activity was calculated as total test time minus time spent immobile in center. Measures were recorded manually by stopwatch.

\section{Open field test}

The open field test allows rapid assessment of rodent locomotion, anxiety and habituation without a training requirement (Hall 1934). The open field apparatus, a Plexiglas square arena of $39 \times 39 \times 38 \mathrm{~cm}$ was designed as a large, brightly lit, open and aversive environment. Locomotor and other activity over a $1 \mathrm{~h}$ period was digitally recorded and scored using Ethovision for distance traveled, velocity and total time in periphery $(10 \mathrm{~cm}$ adjacent to wall $)$ and center.

\section{RNA extraction from brain micropunches}

At sacrifice, using isoflurane anesthesia and cervical dislocation, whole brains were rapidly dissected and snap frozen in 2-methylbutane over dry ice. Brains were cryosectioned ( $0.3 \mathrm{~mm}$ thick) coronally and sections mounted on sterile glass slides and stored at $-80^{\circ} \mathrm{C}$. Five regions of interest were punched out bilaterally from tissue sections under a stereomicroscope using a microdissection blunted needle (16-gauge) adapted from the Palkovits micropunch technique (Palkovits 1973). The anatomical precision was determined by comparing cryosections with cresyl violet stained sections of reference mouse brains and corresponding anatomical plates in the atlas of Paxinos and Franklin. Tissue punches were immediately homogenized in TRIzol Reagent (Thermo Fisher Scientific, USA) using a hand-held homogenizer. Total RNA was prepared via a modified partial phenol-methanol extraction protocol (RNeasy Micro Kit, Qiagen, USA). Purity and quantity of RNA were assessed by determining the optical density (OD) photometrically using 260/280 $\mathrm{nm}$ and 260/230 nm ratios (NanoDrop ND-2000, Thermo-Fisher Scientific Inc., Waltham, MA, USA). RNA integrity was assessed using an Agilent 2100 Bioanalyzer (Agilent Technologies Inc. Santa Clara, CA, USA) (Supplementary data 2).

\section{Quantitative polymerase chain reaction (RT-qPCR) analysis of brain micropunches}

RT-qPCR was used to quantitate mRNA transcripts for pro-social peptides, AVP, OXT, PACAP and their receptors. Custom- or predesigned DNA oligonucleotide PCR primers were obtained from Integrated DNA Technologies.
Primers were designed to meet several criteria using NCBI Primer Blast and then optimized by testing against complementary DNA generated using RT-PCR and gel electrophoresis. Only primers that gave single-band amplicons in the presence of RT and that matched the base length of the predicted target were selected. In addition, primers selected yielded $89 \%$ to $107 \%$ efficiency on RT-qPCR (Table 1). Oxtr and the reference gene, ActB, were multiplexed using hydrolysis probes with double-quenchers. For all other primers, intercalating dye chemistry was used. RT-qPCR was performed on RNA (1-4 ng) samples, run in triplicate, on a CFX Connect (Bio-Rad, USA) thermocycler with the Luna Universal or Probe one-step qPCR Master Mixes (New England Biolabs, Ispwich, MA). In each experiment, no-template controls (NTCs) without mRNA were run to rule out extraneous nucleic acid contamination and primer dimer formation. Negative RT controls, which contained the complete RNA synthesis reaction components without the addition of the enzyme reverse transcriptase (RT) were used to rule out presence of genomic DNA (gDNA). Fold-change gene expression was measured relative to the reference gene, $A c t B$, and differential gene expression was determined compared to null group (VEH/CON) using the Pfaffl method (Pfaffl 2001). Molecular work was carried out in adherence to MIQE guidelines (Bustin et al. 2009) (Supplementary data 2).

\section{Enzyme immunoassays of oxytocin and arginine-8-vasopressin}

Blood was collected by cardiac puncture and the plasma separated at $2000 \times \mathrm{g}$ centrifugation for $20 \mathrm{~min}$ at $4^{\circ} \mathrm{C}$. Plasma levels of the neuropeptides OXT and arginine8-vasopressin were quantified using commercially available ELISA kits from Arbor Assays (Ann Arbor, MI USA; OXT, K048-H1, Arg-8-Vasopressin, K049-C1) and Enzo Life Sciences (Farmingdale, NY, USA; OXT, ADI901153A0001; Arg8-Vasopressin, ADI-900-017) following the manufacturer's instructions. For the Arbor Assay kits, samples were first treated using the acetonebased extraction solution followed by vacuum lyophilization of the resulting supernatant at $37^{\circ} \mathrm{C}$ to reduce the non-specific binding. For oxytocin, the colorimetric reaction product was read as optical density at $450 \mathrm{~nm}$ on a plate reader (SpectraMax 190, Molecular Devices). The kit has a sensitivity of $1.7 \mathrm{pg} / \mathrm{sample}$ in a dynamic range of $16.38-10,000 \mathrm{pg} / \mathrm{mL}$. Arg8-Vasopressin was detected using a luminescence plate reader (Victor3, Perkin Elmer). The Arg8-vasopressin kit has a sensitivity of $0.9 \mathrm{pg} / \mathrm{mL}$ in a dynamic range of $1.638-1,000 \mathrm{pg} / \mathrm{mL}$. For the Enzo Life Sciences kits, samples underwent solid phase extraction using $200 \mathrm{mg} \mathrm{C} 18 \mathrm{Sep}-$ pak columns as previously 
Table 1 RT-qPCR primers and target genes

\begin{tabular}{|c|c|c|c|c|c|c|c|c|}
\hline Target gene & Gene symbol & $\begin{array}{l}\text { GenBank accession } \\
\text { number }\end{array}$ & $\begin{array}{l}\text { Primer/probe } \\
\text { sequence }\end{array}$ & $\begin{array}{l}\text { Exon location } \\
\text { Fwd/Rv }\end{array}$ & $\mathrm{E}(\%)$ & $\operatorname{Tm}\left({ }^{\circ} \mathrm{C}\right) \mathrm{Fwd} / \mathrm{Rv}$ & $\begin{array}{l}\text { Product } \\
\text { size } \\
\text { (bp) }\end{array}$ & $\begin{array}{l}\text { Anneal } \\
\text { temp } \\
\left({ }^{\circ} \mathrm{C}\right)\end{array}$ \\
\hline Arginine vasopressin & $A v p$ & NM_009732.2 & $\begin{array}{l}\text { F: CTCAACACT } \\
\text { ACGCTCTCCGC } \\
\text { R: CAGCAGATG } \\
\text { CTTGGTCCGA }\end{array}$ & $1 / 1-2$ & 98 & $60.8 / 57.9$ & 173 & 55 \\
\hline $\begin{array}{l}\text { Arginine vasopres- } \\
\text { sin receptor 1A }\end{array}$ & Avplar & NM_16847.2 & $\begin{array}{l}\text { F: GCTGGACAC } \\
\text { CTTTCTTCA } \\
\text { TCGTC } \\
\text { R: CTGTTCAAG } \\
\text { GAAGCCAGT } \\
\text { AACG }\end{array}$ & $1 / 2$ & 89.1 & $61.7 / 59.5$ & 115 & 55 \\
\hline $\begin{array}{l}\text { Adenylate cyclase } \\
\text { activating polypep- } \\
\text { tide } 1\end{array}$ & Adcyapl & NM_009625.3 & $\begin{array}{l}\text { F: AGGTGCTGG } \\
\text { TGTTGGAAT } \\
\text { GAATGC } \\
\text { R: AATGCATGA } \\
\text { GGGCAAGGG } \\
\text { TAGGAA }\end{array}$ & 5 & 95 & $60.2 / 60.7$ & 176 & 55 \\
\hline $\begin{array}{l}\text { Adenylate cyclase } \\
\text { activating polypep- } \\
\text { tide } 1 \text { receptor } 1\end{array}$ & Adcyap1r1 & NM_007407.4 & $\begin{array}{l}\text { F: TTCACTACT } \\
\text { GCGTGGTGT } \\
\text { CCAACT } \\
\text { R: ATATCCCAG } \\
\text { CATCCCGCA } \\
\text { TCATCA }\end{array}$ & $10 / 11-12$ & 96.3 & $60.3 / 60.3$ & 199 & 55 \\
\hline Oxytocin & Oxt & NM_011025.4 & $\begin{array}{l}\text { F: CCGAAGCAG } \\
\text { CGTCTTTT } \\
\text { R: CTTGGCTTA } \\
\text { CTGGCTCTGAC }\end{array}$ & $1 / 2$ & 96.9 & $55.7 / 55.5$ & 131 & 60 \\
\hline Oxytocin receptor & Oxtr & NM_001081147.2 & $\begin{array}{l}\text { F: CGCACAGTG } \\
\text { AAGATGACCTT } \\
\text { R: ATGGCAATG } \\
\text { ATGAAGGCAGA } \\
\text { P: 6-FAM-CTTCGT } \\
\text { GCA-ZEN-GAT } \\
\text { GTGGAGCGT } \\
\text { TCT-IBFQ }\end{array}$ & $1 / 2$ & 107.1 & NA & 131 & 60 \\
\hline Beta actin & $\beta$-Actin & NM_007393.5 & $\begin{array}{l}\text { F: GATTACTGC } \\
\text { TCTGGCTCC } \\
\text { TAG } \\
\text { R: GACTCATCG } \\
\text { TACTCCTGC } \\
\text { TTG } \\
\text { P: HEX-CTGGCC } \\
\text { TCA-ZEN-CTG } \\
\text { TCCACCTTC } \\
\text { C-IBFQ }\end{array}$ & $5 / 6$ & $\begin{array}{l}99.6 \\
101.7\end{array}$ & $\begin{array}{l}55.0 / 54.4 \\
\text { NA }\end{array}$ & 147 & 60 \\
\hline
\end{tabular}

Abbreviations: $F$ forward, $R$ reverse, $P$ probe, $E$ primer efficiency, $T m$ melting temperature, $b p$ base pair, ZEN/IBFQ ZEN-Iowa Black FQ, FAM Fluorescein, HEX Hexachloro-fluorescein

described (Deol et al. 2020). Plasma oxytocin and arginine vasopressin were quantified by interpolating absorbance or luminosity values, respectively, using a 4-parameterlogarithmic standard curve (MyAssays).

\section{Statistical analyses}

Statistical analysis was performed using GraphPad Prism (version 8.4.3 San Diego, CA, USA). Within group comparisons were performed using paired Student's t test. Between groups comparisons were accomplished using one-way, twoway or mixed model ANOVA with or without a repeated measures design. Non-parametric statistical tests (i.e., 
Kruskal-Wallis H test) were used when normality and/or equal variances assumptions were not met as measured using the Shapiro-Wilk and F-tests. If an equal variance assumption was not met, a Brown-Forsythe ANOVA or Welch's correction was used. Post hoc comparisons were performed using appropriate tests. Biological outliers were excluded when animals were unable to perform behavioral tests. Type 1 error rate $(\alpha)$ was set at $0.05 ; \mathrm{F}$ and $P$ values are presented in the figure legends or Supplemental statistical information. The data are expressed as the mean \pm s.e.m, in bar or scatter plots or as median and inter quartile range representing minimum and maximum values in whisker plots.

\section{Results}

\section{DE-71 dosing paradigm and maternal parameters}

C57B1/6 mice dams were exposed to DE-71 and later investigated along with the F1 female offspring as shown in diagram (Fig. 1). Using this dosing paradigm, we have previously reported no differences in litter size at birth, secondary sex ratio, nor gestational maternal parameters (Kozlova et al. 2020). Moreover, dams exposed to DE-71 did not build inferior nests and F1 litters at PND 46 had normal body mass relative to VEH/CON (Supplementary Fig. 1). In combination, these data indicate that perinatal DE-71 exposure does not interfere with pup health, maternal nest quality nor related behaviors shown to be affected by exposure to PCBs, a structural/functional analogue class of PBDEs (Abu-Arafeh et al. 2016).

\section{PBDE congener analysis in offspring brain}

PBDE congener content was determined using HRGC/ HRMS or GC/ECNI-MS in F1 female brain from offspring during the lactational period (PND 15) or as adults (PND 110), respectively. Raw values are listed by exposure group in Supplementary Tables 3, 4, 5. Figure 1 b,c show a significant increase in $\sum$ PBDEs in L-DE-71 and H-DE-71 relative to $\mathrm{VEH} / \mathrm{CON}(P<0.05)$, confirming that the dosing regimen led to maternal transfer of PBDEs to offspring brain. Accumulation of PBDEs in PND 15 (but not PND 110) was dosedependent $(P<0.05)$. Mean $\sum_{14}$ PBDE values in exposed $\mathrm{F} 1$ at PND 15 were 78 and $296 \mathrm{ng} / \mathrm{g} \mathrm{w.w}$. for L-DE-71 and $\mathrm{H}-\mathrm{DE}-71$, respectively. The corresponding mean values of total PBDEs (of which only BDE-153 was above detection limits) at PND 110 were 0.53 and $1.5 \mathrm{ng} / \mathrm{g}$ w.w. and 113 and $169 \mathrm{ng} / \mathrm{g}$, respectively, when normalized to lipid weight (1.w.). For PND 15 the composition of BDEs in L-DE-71 and $\mathrm{H}-\mathrm{DE}-71$ were (in \%): BDE-17 (0.021 and 0.006\%),
BDE-28 (0.088 and 0.126\%), BDE-47 (12.2 and 17.4\%), BDE-49 (0.014 and 0.017\%), BDE-85 (1.63 and 1.41\%), BDE-99 (36.3 and 34.0\%), BDE-100 (12.2\% and $11.3 \%)$, BDE-138 (0.572 and 0.488\%) BDE-139 (2.90 and 2.70\%), BDE-140 (0.408 and 0.288), BDE-153 (30.2 and 29.5\%), BDE-154 (3.12 and 2.48), BDE-183 (0.244 and 0.168), BDE-184 (0.185 and 0.182\%), respectively (Fig. 1d). Collectively, seven congeners (BDE-47, -85, -99, -100, -139, $-153,-154)$ in L-DE-71 and H-DE-71 accounted for 98.5 and $98.7 \%$, respectively, of all PBDEs penetrating the brain during lactation. These same seven congeners comprise $97.1 \%$ of the DE-71 mixture. The remaining 7 of 14 congeners detected in our samples, made up the remaining 1.5 and $1.3 \%$, respectively: BDE-17, 28, 49, 138, 140, 183 and 184. Figure 1e shows that, with the exception of BDE-17, 28, 49 and 184 in L-DE-71 and BDE-49 in H-DE-71, all 14 congeners detected showed significantly elevated concentrations in DE-71 exposed offspring at PND 15 relative to VEH/CON $(P<0.05$ and $P<0.01)$. Of note, BDE-153 was $\sim$ tenfold enriched and BDE-47 was slightly depleted ( $\sim$ twofold) relative to the DE-71 mixture as reported previously (Kodavanti et al. 2010).

By PND 110, the BDE composition in F1 brain was limited to BDE-153 (Fig. 1f), which was significantly elevated in L-DE-71 and H-DE-71 relative to VEH/CON $(P<0.01$ and $P<0.05$ ). In comparison, BDE-153 at ppb (and an additional 6 congeners) has been reported in postmortem brain samples from 4-71 year-old neurotypical controls and autistic humans born in 1940-2000 (Mitchell et al. 2012).

\section{Early-life exposure to DE-71 induces deficits relevant to core symptoms of autism in F1 female progeny}

Social novelty preference. Testing mice on a social novelty preference (SNP) test has been suggested to be ethologically relevant to social domains affected in autistics (Moy et al. 2004). On this test, all F1 exposure groups except the L-DE-71 F1 group $(P<0.05)$ showed a preference for the novel over familiar stimulus (Fig. 2a), and this was also represented in the recognition index vs VEH/CON (Fig. 2b, $P<0.05)$. In contrast, there was no effect of exposure in F0; all groups showed a preference for novel stimulus (Fig. 2c, $P<0.0001)$ and no group differences were observed in the recognition index (Fig. 2d). The investigation index for F1 and F0 groups approached 1 (Supplementary Fig. 2) indicating that the reduced exploration of novel over familiar shown by L-DE-71 F1 was not due to a decrease in total investigation time indicating no lack of participation.

Sociability. To determine social interest, an independent social cognition domain, we examined mouse behavior 

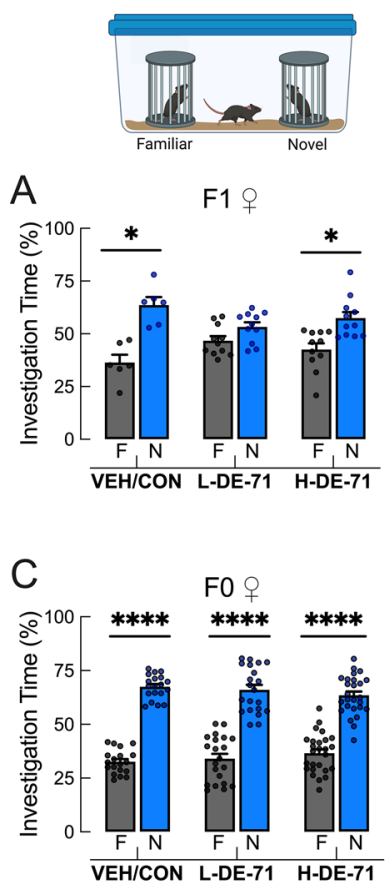

I

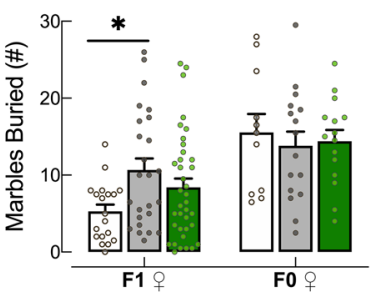

B

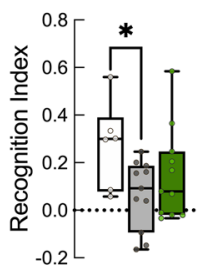

Social Novelty

Preference
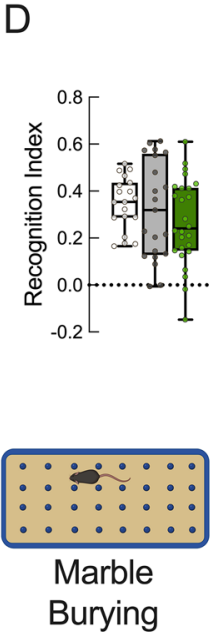

E

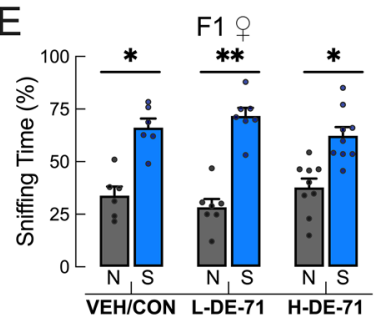

$\mathrm{F}$

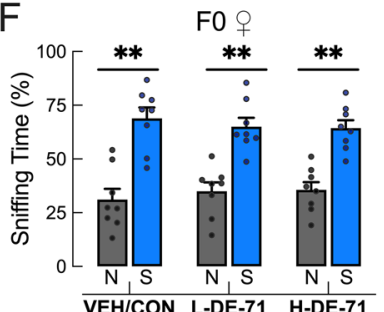

Sociability

VEH/CON L-DE-71 H-DE-71

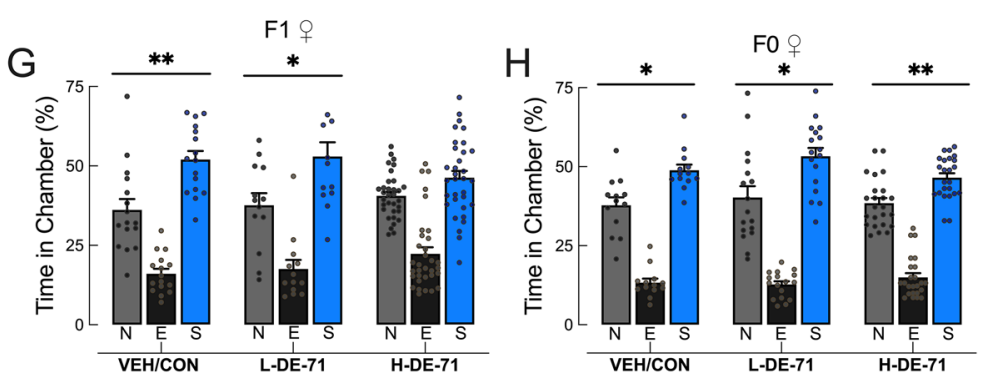

$J$

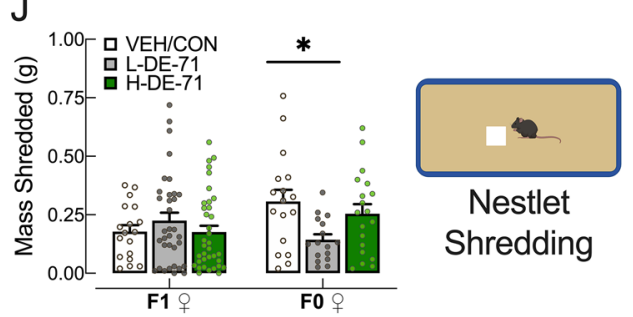

Fig. 2 Early-life exposure to DE-71 induces deficits relevant to core symptoms of ASD in F1 female progeny. a, c Social Novelty Preference scores for dams and female offspring: unlike the F1 VEH/CON and F1 H-DE-71, F1 L-DE-71 females failed to spend more time with a novel relative to a familiar conspecific stimulus. F0 dams exposed to DE-71 did not show abnormal social recognition relative to VEH/ CON. b, d Recognition Index scores show decreased preference for novel stimulus in L-DE-71 F1 relative to VEH/CON but not in F0. e, $\mathbf{f}$ Time spent sniffing in sociability test. All exposure groups spent significantly more time sniffing social stimulus indicating normal sociability. $\mathbf{g}$, h Chamber time scores in sociability. All groups show significantly greater time spent in the social chamber relative to non- social except for F1 H-DE-71. i Marble burying scores showed offspring L-DE-71 buried a greater amount of marbles as compared to VEH/CON and H-DE-71, but not in dams. j Nestlet shredding was not affected in exposed $\mathrm{F} 1$ but was reduced in L-DE-71 F0 relative to corresponding $\mathrm{VEH} / \mathrm{CON}$. $* P<.05, * * P<.01 ; * * * * P<.0001$ compared to VEH/CON (b,d,i,j), familiar $(\mathrm{a}, \mathrm{c})$ or non-social chamber (e,f,g,h). $n=6-11$ litters/group (a-b), 19-26 subjects/group (c-d), 6-9 litters/group (e), 8 litters/group (f), 16-33 subjects/group (g), 13-24 subjects/group (h), 19-37 subjects/group for F1 and 11-16 subjects/group for F0 (i), 18-36 subjects/group for F1 and 16-19 subjects/group for F0 (j). $F$ familiar, $N$ novel, $N$ non-social, $S$ social, $N$ non-social, $E$ empty, $S$ social on a 3-chamber sociability test. All F1 groups (VEH/CON, L-DE-71, H-DE-71) showed preference for a novel social stimulus relative to a non-social novel stimulus as measured by sniffing time (Fig. 2e, $P<0.05,0.01,0.05$, respectively), indicating normal sociability. Using chamber time VEH/ CON and L-DE-71, but not H-DE-71 F1, showed a preference for social stimulus (Fig. $2 \mathrm{~g}, P<0.05, P<0.01$, ns, respectively). Sniffing time has been suggested to have superior validity over chamber time scores since active behaviors that are most directly related to social investigation are captured (Fairless et al. 2011) and the physical proximity allows for transmission of volatile and nonvolatile oderants (Brennan and Kendrick 2006); (Luo et al. 2003); (Fairless et al. 2011). For F0, chamber (Fig. 2f, $P<0.01$ ) and sniffing time scores inidcated a preference for social stimulus and no effect of exposure was found (Fig. $2 \mathrm{~h} P<0.05$ for VEH/ CON and L-DE-71, and $P<0.01$ for H-DE-71). As a measure of test robustness there was no indication of side preference during training for F1 and F0 (Supplementary Fig. 2).

Repetitive behavior. On the marble burying test, which measures repetitive and perseverative behavior in rodents (Thomas et al. 2009), L-DE-71 (but not H-DE-71) adult F1 
buried a significantly greater number of marbles relative to VEH/CON (Fig. 2i, $P<0.05$ ). A subgroup of F1 was tested at PND 30, but no group differences were detected, possibly indicating age-related physical hypoactivity, reduced habituation to the test arena or a latently emerging impact of PBDEs (Supplementary Fig. 3). Again, no group differences were seen in F0 (Fig. 2i). Mean values for nestlet shredding were not affected by DE-71 exposure in F1. However, in F0, the L-DE-71 group showed a mean reduction in nestlet shredding relative to VEH/CON (Fig. 2 j, $P<0.05$ ). However, less nestlet shredding did not translate into poorer maternal nest scores (Supplementary Fig. 1).

\section{Exposure to L-DE-71 but not H-DE-71 reduces long-term social recognition memory in F1 female progeny}

We determined that SNP scores requiring a 30 min memory retention were abnormal in exposed F1 but not F0. To test the hypothesis that DE-71 compromises consolidation of long-term social recognition memory, we subjected F1 to a social recognition memory test (SRMT) (Tanimizu et al. 2017). On this test, mice with intact memory exhibit less time investigating a familiar juvenile conspecific $24 \mathrm{~h}$ after a first exposure. Figure 3 a shows that VEH/CON and H-DE71 mice were able to form a social recognition memory of the stimulus by Day 2 since they spent significantly less time with a familiar stimulus mouse $(P<0.05$ and $P<0.0001$, respectively). Mean RI values for F1 were no different for VEH/CON $(0.71)$ or less for H-DE-71 $(0.56, P<0.05)$, suggesting no deficits in recognition memory (Fig. 3b). In contrast, L-DE-71 F1 showed an apparently greater RI (mean RI, $0.85, P=0.07$ ), indicating potentially deficient long-term social recognition memory (Fig. 3b). Next, we examined investigation time with a second novel stimulus mouse on Day 2 to determine whether the reduction of investigation time on Day 2 is specific to social memory formation and not due to disengagement. In Fig. 3c, no significant reduction of investigation time was noted as expected for $\mathrm{VEH} / \mathrm{CON}$ and H-DE-71, suggesting that the reduction in investigation of familiar mouse above was specific to recognition memory formation. In contrast, L-DE-71 F1 exhibited a significant reduction in investigation time of new novel mouse on Day $2(P<0.05)$, indicating deficient social memory. In Fig. 3d L-DE-71 showed a significantly lower RI than $1(0.89$, $P<0.05)$. During test optimization using untreated controls, we confirmed that our test was valid, i.e., that the 3 but not $1 \mathrm{~min}$ of social exposure on Day 1 was sufficient to form a memory on Day $2(P<0.01)$ as reported (Tanimizu et al. 2017) (Supplementary Fig. 4). In summary, these results indicate that developmental exposure to DE-71 at $0.1 \mathrm{mg} /$ $\mathrm{kg} / \mathrm{d}$ but not $0.4 \mathrm{mg} / \mathrm{kg}$ significantly reduces long-term social recognition memory in F1.
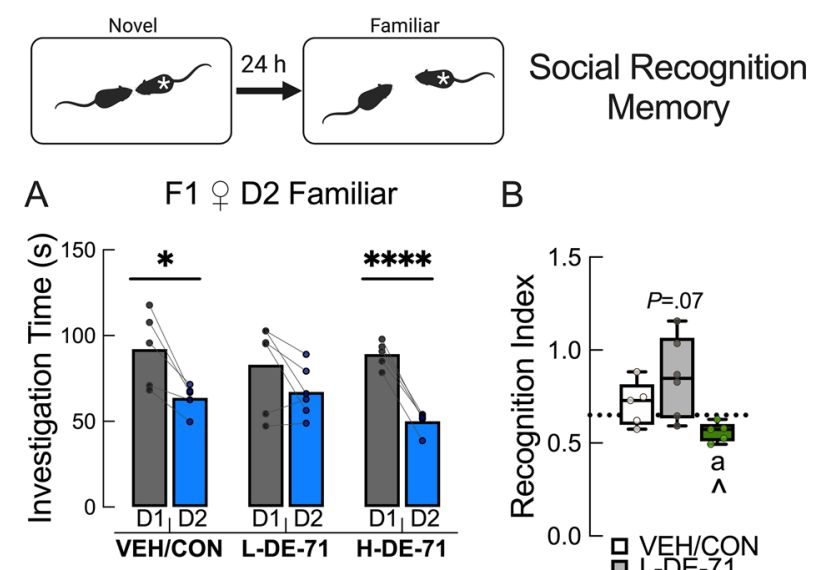

B
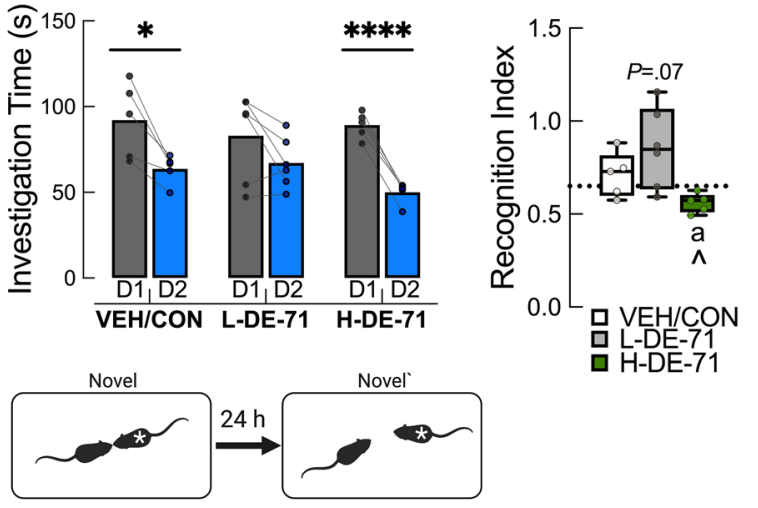

C F1 + D2 Novel'

D

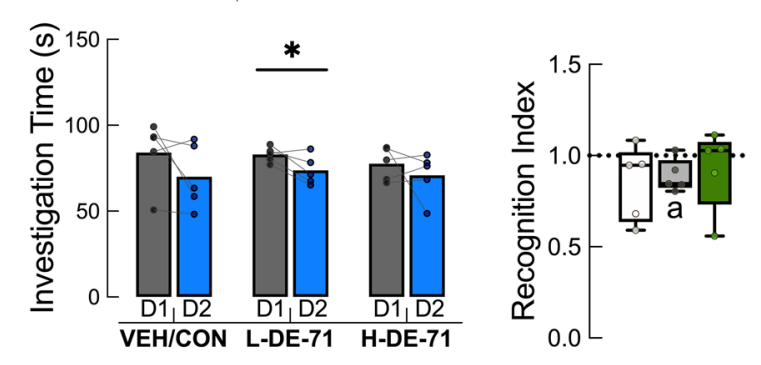

Fig. 3 Exposure to L-DE-71 but not H-DE-71 reduces long-term social recognition memory in $\mathrm{F} 1$ female progeny. a When using a familiar stimulus the VEH/CON and H-DE-71 F1 mice displayed a significant reduction in investigation time on Day 2, indicating normal SRM. In contrast, L-DE-71 showed no reduction in investigation after the $24 \mathrm{~h}$ retention period. b Scores for Recognition Index (RI), representing reduction in investigation of familiar mouse on Day 2 relative to Day 1 , indicate normal social recognition memory since mean scores were no different from 0.65 for $\mathrm{VEH} / \mathrm{CON}$ and $\mathrm{H}-\mathrm{DE}$ 71 groups. The mean RI value for L-DE-71 was apparently greater indicating that modest reduction of investigation of familiar mouse on Day 2 may be due to chance. c When using a different novel mouse on Day 2 (Novel'), VEH/CON and H-DE-71 F1 mice showed equal preference for novel mice on Day 2 relative to Day 1, indicating normal recognition memory and that the reduction in investigation time in $a$ and $b$ is specific to familiar juveniles. However, L-DE-71 mice were unable to do this. d Mean RI scores are less than 1 only for L-DE-71, indicating unequal preference for Novel (Day 2) vs Novel stimulus (Day 1) and abnormal social recognition memory. $* P<.05$, $* * * * P<.0001$ compared to Day 1 (a, c). ${ }^{\wedge} P<.05$ compared to L-DE71. ${ }^{\text {a }} P<.05$ compared to .65 (b) or $1.0(\mathbf{d}) . n=5-6$ litters/group. '*', stimulus mouse in insets. $D$ day

\section{Perinatal exposure to L-DE-71 compromises short-term novel object recognition memory in $\mathrm{F} 1$ females and FO}

Having found that DE-71 exposure produces significant impairment in the SNP and SRMT, we tested the 


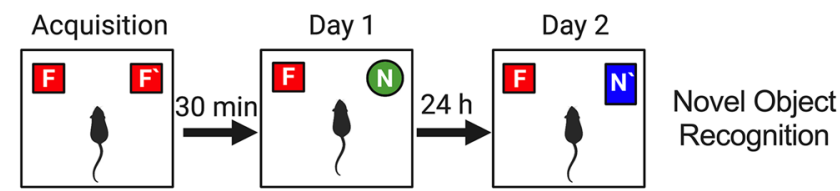

A

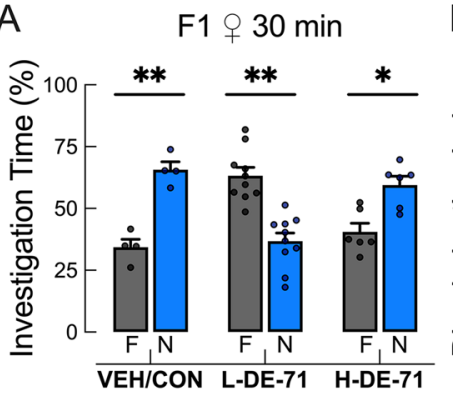

B

C
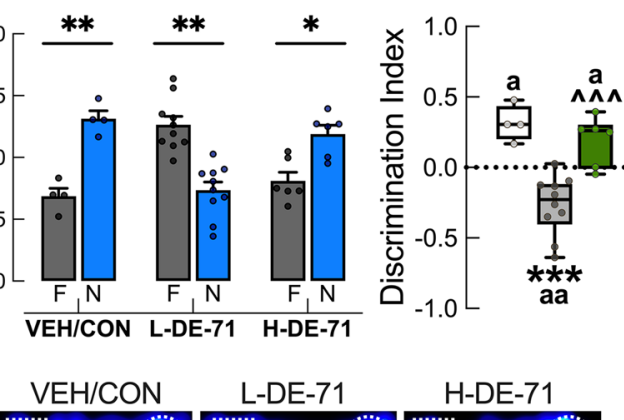

H-DE-71

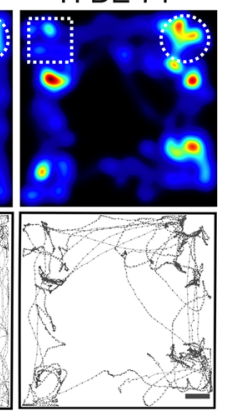

E

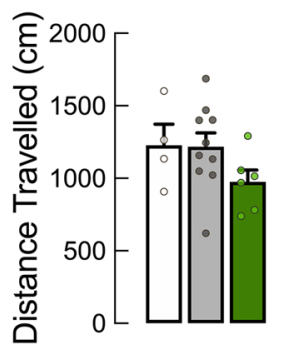

$\mathrm{F}$

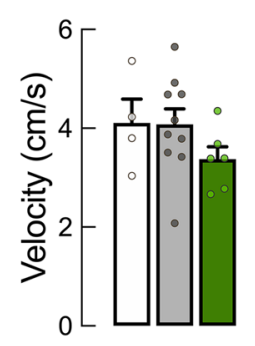

Fig. 4 Perinatal exposure to L-DE-71 compromises short-term novel object recognition memory in F1 females and F0. a Investigation time on the novel object recognition test. F1 offspring in the VEH/ CON and H-DE-71 but not L-DE-71 group show significantly greater time spent investigating the novel (circle, $\mathrm{N}$ ) vs familiar (square, F). b L-DE-71 F1 shows a significant negative discrimination index indicating less time spent with novel object. c Representative dwell-time maps (triple gradient, blue-minimum; green-intermediate red-maximum) of time spent exploring novel and familiar objects showed differences in dwell times for different exposure groups. $\mathbf{d}-\mathbf{f}$ Representa-
G

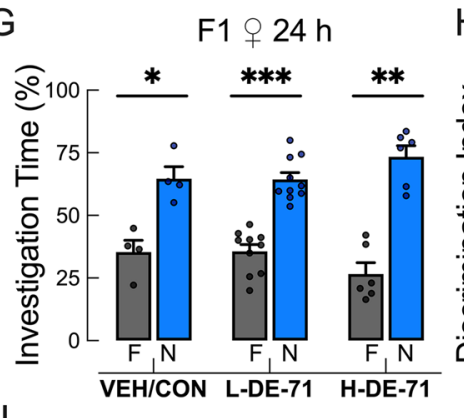

$\mathrm{H}$

I

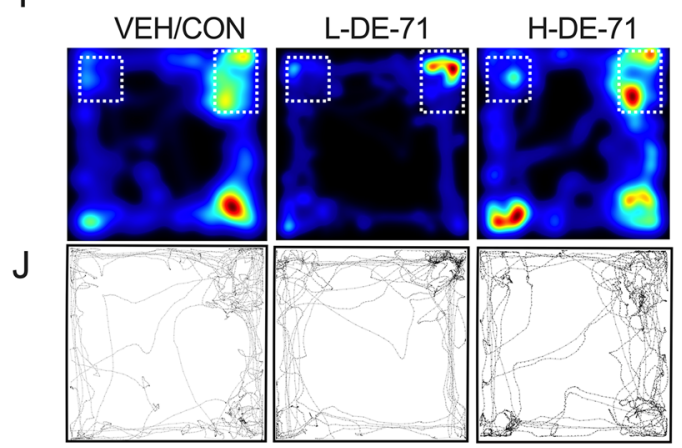

$\mathrm{K}$

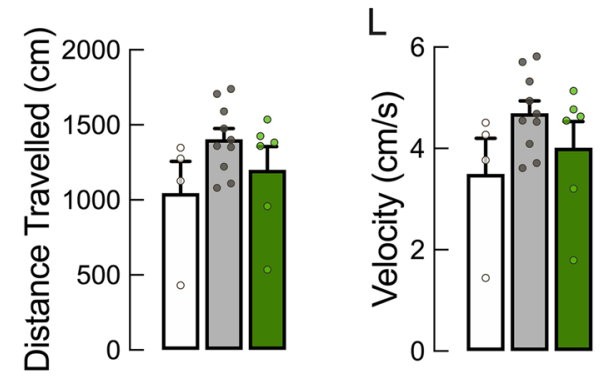

tive raster plots indicate no significant effect of exposure on general locomotor activity quantified as cumulative distance travelled and velocity. g-l After a $24 \mathrm{~h}$ retention time there was no effect of exposure on investigation time of familiar and novel, discrimination index, dwell-time maps, raster plots, distance travelled, or velocity. $* P<.05$, $* * P<.01, * * * P<.001$ compared to familiar object (a) or VEH/ CON (b). ${ }^{\wedge \wedge \wedge} P<.001$ compared to L-DE-71 (b). a $P<.05$, ${ }^{\text {aa }} P<.01$, ${ }^{\text {aaa }} P<.001$ compared to $0(\mathrm{~h}) . n=4-10$ subjects/group. $F$ and $F$, familiar object; $N$ and $N^{`}$, novel object. Scale bar, $5 \mathrm{~cm}$

recognize objects presented 30 min earlier in the familiarization phase. This was corroborated using a discrimination index which showed that values for only VEH/ $\mathrm{CON}$ and $\mathrm{H}-\mathrm{DE}-71$ were $>0$, indicating memory for previously encountered objects (Fig. $4 \mathrm{~b}, P<0.05$ ). In contrast, L-DE-71 group displayed a negative mean discrimination 
index (greater preference for familiar object, $P<0.01$ ), a result that was significantly reduced compared to VEH/ CON $(P<0.001)$. Representative dwell time maps in the open field arena showed preference for novel object (right corner) for VEH/CON and H-DE-71 on Day 1 (Fig. 4c). In contrast, L-DE-71 showed less exploration of novel relative to familiar object. On Day 2 all exposure groups preferred novel over familiar object and showed similar mean values for discrimination index and dwell times after a $24 \mathrm{~h}$ retention time (Fig. $4 \mathrm{~g}, \mathrm{~h}, \mathrm{i}, \mathrm{j}$ ). There were no effects of exposure on mean distance travelled and velocity (Fig. 4e, f, k, l) as indicated by raster plots (Fig. 4d, j). Interestingly, both L-DE-71 and H-DE-71 exposed dams showed similar short-term memory deficits as L-DE-71 exposed F1 offspring (Supplementary Fig. 5).
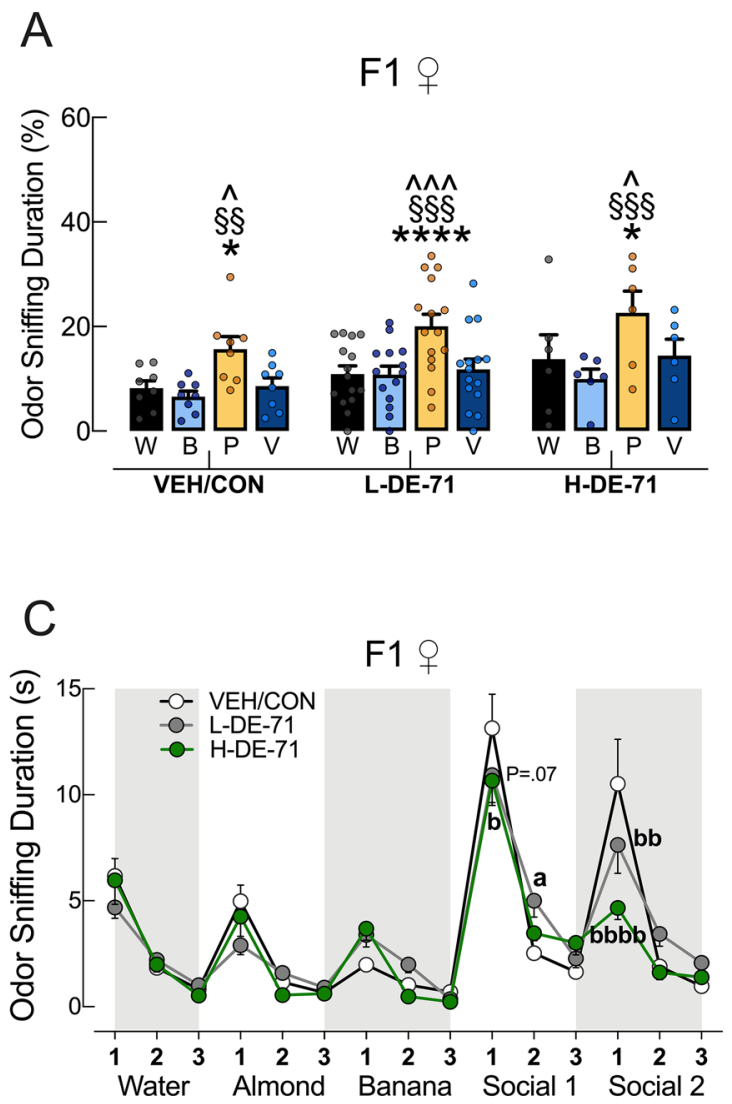

Fig. 5 Perinatal exposure to DE-71 does not alter general olfaction function but disrupts discrimination of social odors. a, b Olfactory preference test on F1 and F0. Both groups showed normal olfactory preference for peanut butter odor. c, d Sniffing time on Olfactory habituation/dishabituation test showed that relative to VEH/ CON, L-DE-71 F1 mice showed less habituation to social odor 1 and 2. Both L-DE-71 and H-DE-71 showed abnormally reduced dishabituation to social odor 2. H-DE-71 showed reduced dishabituation to social odor 1 , an effect that was apparent in L-DE-71.

\section{Abnormal social behavior in F1 produced by DE-71 exposure is not due to deficits in general olfactory processing}

To examine if DE-71-induced deficits observed in social recognition ability were due to insufficient olfactory ability, we subjected female offspring to an olfactory preference test. Figure 5a shows that all mice including those treated with DE-71 displayed increased odor sniffing duration for peanut butter over water $(P<0.05-0.0001)$, butyric acid $(P<0.05-0.0001)$, and vanilla $(P<0.05-0.001)$. Similar results were obtained for dams (Fig. 5b). These results indicate that, like VEH/CON, DE-71 exposed offspring and dams were able to process sensory signals from different non-social odors with enough sensitivity to show preference for peanut butter over others.
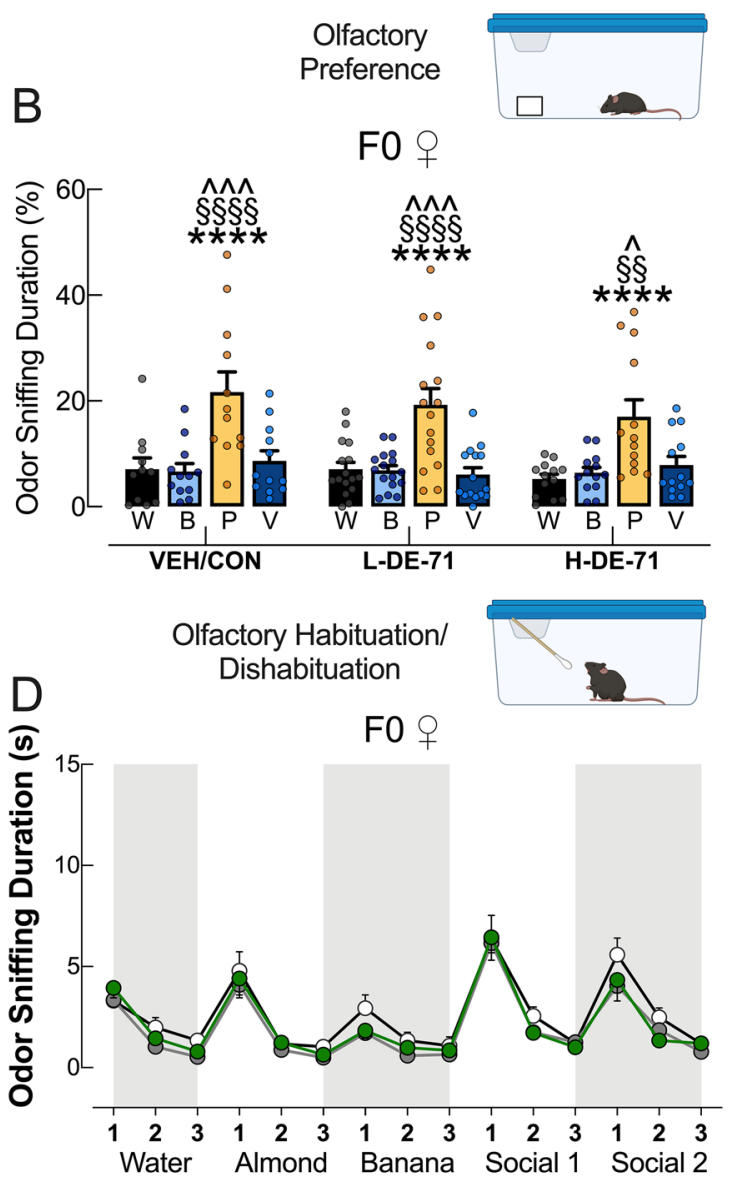

No group differences were noted for $\mathrm{F} 0 . * P<.05$, $* * * * P<.0001$ compared to water. ${ }^{\wedge} P<.05,{ }^{\wedge} \wedge P<.001$ compared to vanilla; $\S \S P<.01, \quad \S \S \S P<.001, \quad \S \S \S \S><.0001$ compared to butyric acid. ${ }^{\mathrm{a}} P<.05$, ${ }^{\text {aaa }} P<.001$ compared to $\mathrm{VEH} / \mathrm{CON}$ during habituation. ${ }^{\mathrm{b}} P<.05,{ }^{\mathrm{bb}} P<.01,{ }^{\mathrm{bbbb}} P<.0001$ compared to $\mathrm{VEH} / \mathrm{CON}$ during dishabituation. Additional statistical results are summarized in Table 2. $n=6-15$ litters/group (a), $n=11-16$ subjects/group (b), $n=12-16$ subjects/group (c), $n=12-16$ subjects/group (d). $W$ water, $B$ butyric acid, $P$ peanut butter, $V$ vanilla 


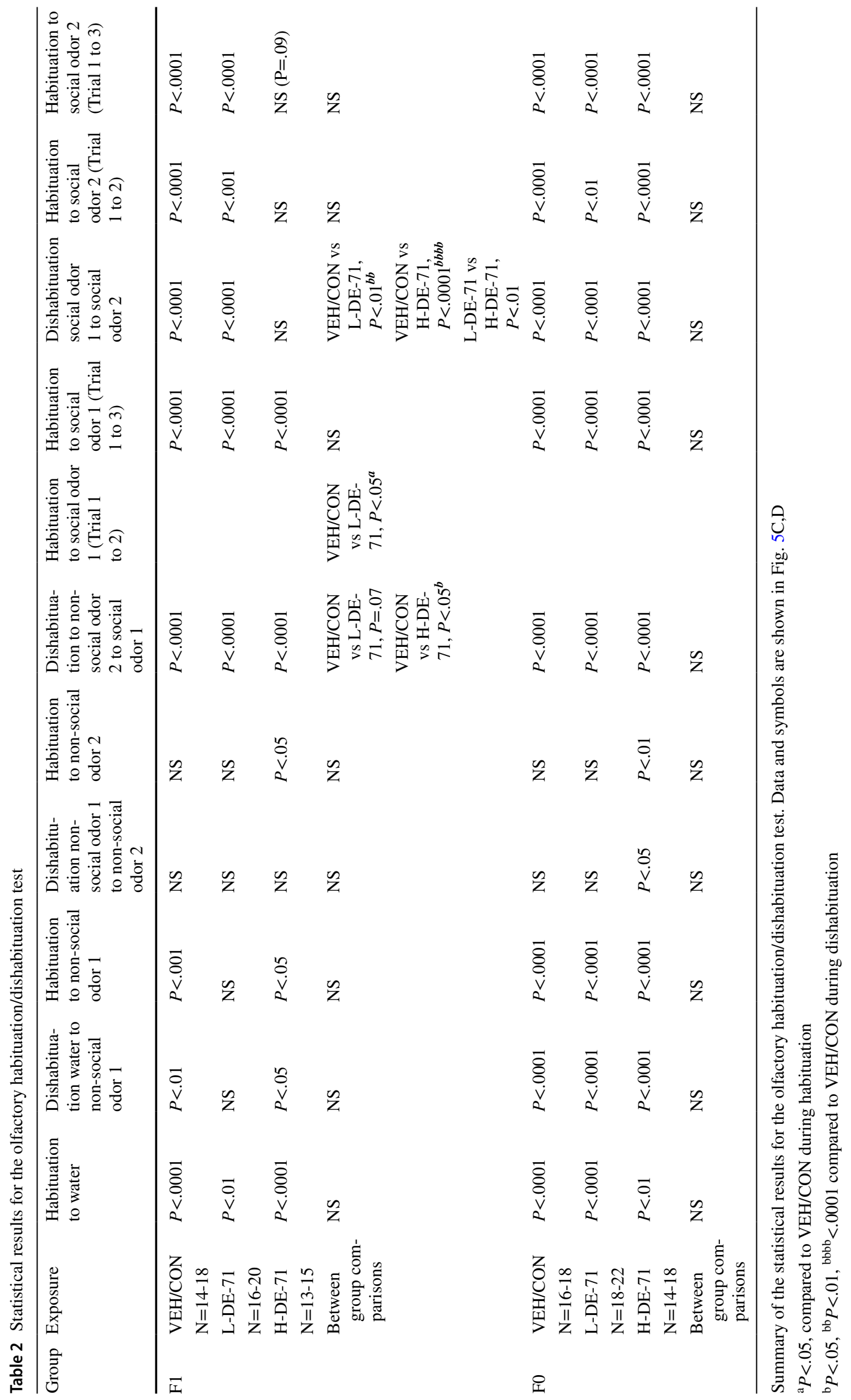




\section{DE-71 exposure alters olfactory discrimination of social odors}

We used an olfactory habituation/dishabituation test to measure olfactory discrimination. Table 2 indicates the results of the habituation/dishabituation test. The F1 VEH/CON group displayed olfactory habituation to all non-social odors and social odors (except non-social odor 2- banana) as indicated by the decline in time spent sniffing by trial 3 (Fig. 5c). F1 VEH/CON displayed olfactory dishabituation when transitioning to a new odor except from non-social 2 (banana) to social $1(P<0.01, P<0.0001)$. Both DE-71 groups displayed deficient habituation and/or dishabituation for more than 1 odor (Table 2). In particular, L-DE-71 showed reduced habituation to social odor 1 (from trial 1 to $2 ; P<0.05$; Fig. $5 \mathrm{c}$ ). Figure $5 \mathrm{c}$ shows that compared to VEH/CON, L-DE-71 and H-DE-71 showed less dishabituation from social odor 1 to $2(P<0.01$, $P<0.0001)$, suggesting that DE-71 produces reduced olfactory discrimination (hyposmia) especially of social odors, which requires processing via MOE and VNO (Huckins et al. 2013). In addition, H-DE-71 also showed reduced dishabituation from non-social odor 2 to social odor $1(P<0.05)$. An apparently significant effect was also seen for L-DE-71 $(P=0.07)$. These findings indicate altered social odor discrimination after perinatal exposure to DE-71 potentially associated with altered signaling through the VNO and less associated with MOE which also processes non-social odors which are normal. Olfactory discrimination of odors in F0 showed normal habituation/dishabituation profiles compared to VEH/CON (Fig. 5d, Table 2). There were no exposure group differences found for F0.

\section{DE-71 exposure does not promote anxiety nor depressive-like behavior}

Mice were evaluated for anxiety using the EPM test and time spent in closed arms relative to open arms was significantly greater in all exposure groups in F1 and F0 $(P<0.0001)$. There was no effect of exposure on the number of total arm entries for F1. In contrast, the H-DE-71 F0 group exhibited significantly fewer total entries relative to VEH/CON (Supplementary Fig. 6). Using a forced swim test, depressive-like behavior was measured as time spent immobile and there was no significant effect of exposure on time spent immobile for F1 nor F0 as compared to VEH/CON (Supplementary Fig. 6).

\section{Selective effects of DE-71 exposure on Suok test}

Using Suok, we measured the effects of DE-71 exposure on locomotion, exploratory behavior, sensorimotor coordination and anxiety. Relative to VEH/CON, H-DE-71 (but not L-DE-71) F1 showed decreased horizontal activity, as represented by segments crossed (Fig. 6a), decreased locomotion (Fig. 6b), decreased exploratory activity (Fig. 6g), increased SAP (Fig. 6i) and decreased grooming (Fig. 6k). Falls were significantly decreased in H-DE-71, but not when normalized to segments crossed (Fig. 6c, d). In contrast to F1, F0 exposed to H-DE-71 showed decreased hind leg slips (Fig. 6e) and L-DE-71 showed increased SAP relative to VEH/CON (Fig. 6i). There were no significant differences on the other measures.

\section{Early-life PBDE exposure does not alter locomotion on the open field test}

The open field test informs about locomotion, habituation to novelty and anxiety. All F1 exposure groups showed similar reduced exploratory activity over time (habituation), measured as reduced distance traveled and velocity over the $1 \mathrm{~h}$ test (Fig. 7 a,b, $P<0.0001$ ). Between-group comparisons showed no effect of exposure for F1 when compared to VEH/CON. These results helped us rule out concerns of hyper- or hypomobility in DE-71 exposed female offspring relative to VEH/ CON as reported after acute exposure to $0.8 \mathrm{mg} / \mathrm{kg}$ BDE-99 at PND 10 (Viberg et al. 2004); (Costa and Giordano 2007). Other studies using chronic exposure of mouse dams to low doses of BDE-47 $(0.1 \mathrm{mg} / \mathrm{kg})$ or $-99(0.6 \mathrm{mg} / \mathrm{kg})$ from gestation through third week of lactation have shown inconsistent results with both hypoactivity and no effect reported on the open field test in female offspring (Ta et al. 2011); (Koenig et al. 2012); (Branchi et al. 2002).

Exploration time in center and periphery zones for all exposure groups (Fig. 7c,d) showed habituation only in the periphery $(P<0.0001)$. Figure 7 e shows that total distance travelled in the periphery was similarly and significantly greater than in center in all exposure groups $(P<0.0001)$. Another measure of anxiety, number of fecal boli at 5 and 10 min into the test, indicated increased emotional reactivity in L-DE-71 F1 relative to VEH/CON (Fig. 7f, $P<0.05, P<0.01$, respectively). For F0 H-DE-71 showed greater distance travelled and velocity in the arena and exploration in the periphery zone as compared to VEH/CON $(P<0.05$ and $P<0.01$, respectively $)$ and L-DE-71 $(P<0.05$ and $P<0.0001)$. L-DE-71 mice produced more fecal boli at 60 min relative to VEH/CON (Supplementary Fig. 7).

\section{DE-71 exposure alters prosocial gene expression in brain regions involved in social behavior}

To correlate the behavioral findings with changes in gene expression of the social neuropeptide systems that are key mediators of complex social behavior, such as vasopressin $(A v p)$, oxytocin $(O x t)$, PACAP (Adcyapl) and their receptors, we measured the relative expression of their genes from micropunches of discrete brain nuclei involved in social behavior: lateral septum, amygdala, bed nucleus of the stria 

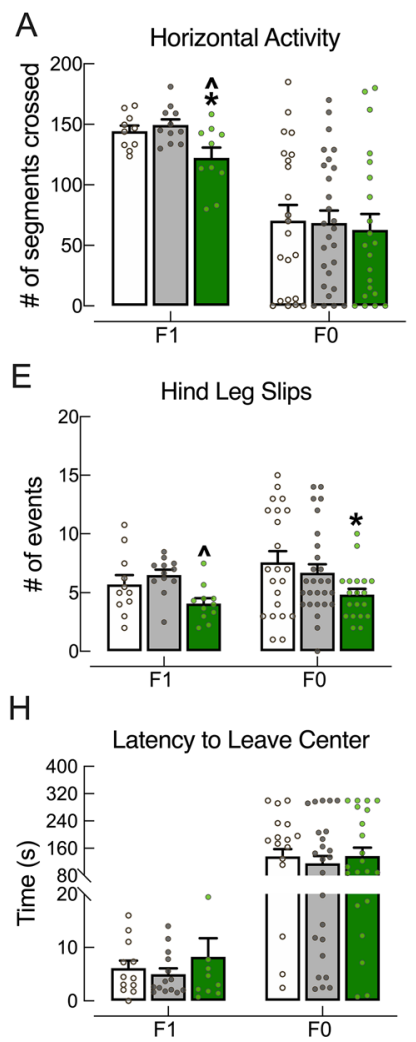

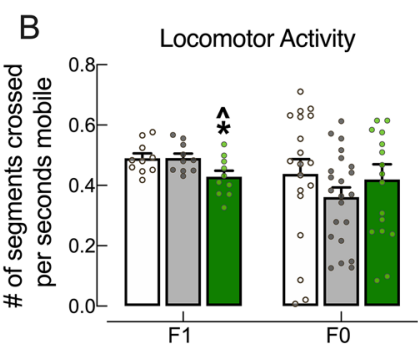

F

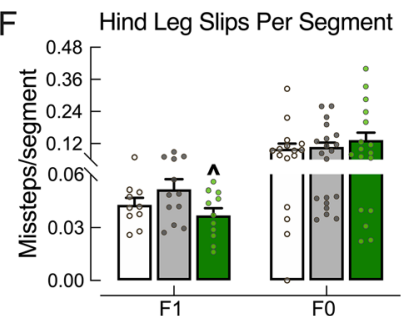

I

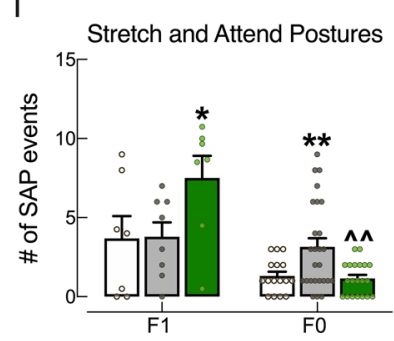

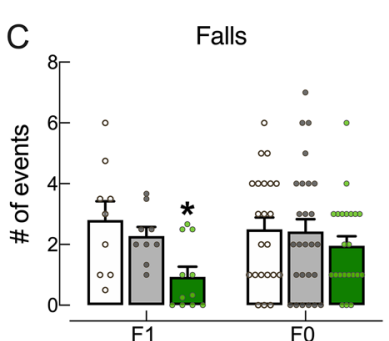
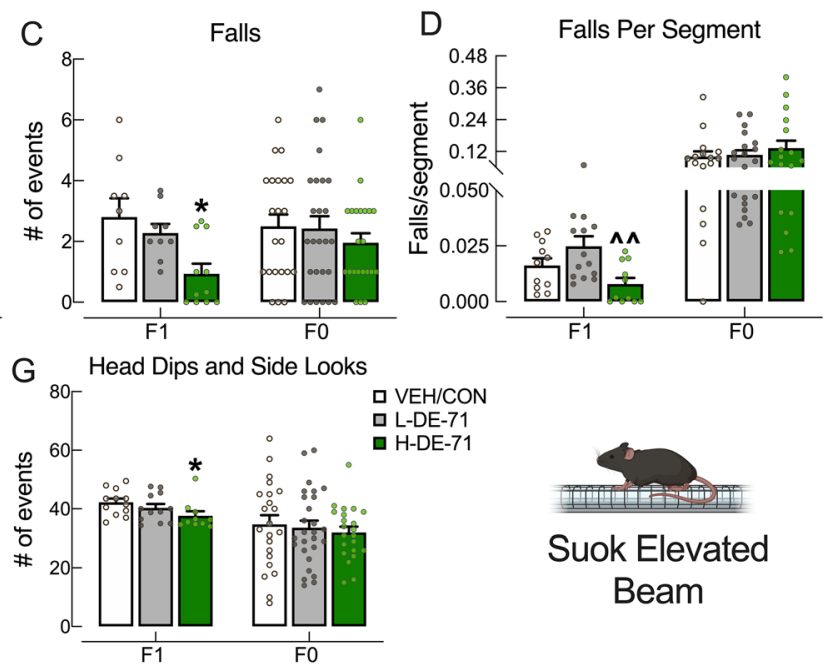

Suok Elevated

Beam
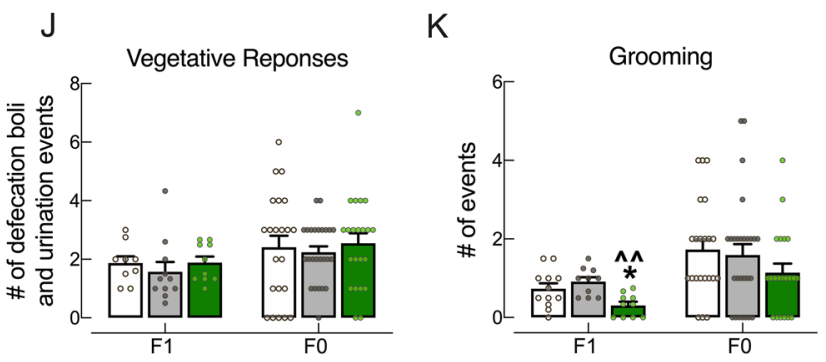

Fig. 6 Selective effects of DE-71 exposure on Suok Test. Female offspring and dams were tested on SUOK for: $\mathbf{a}$, b locomotion; c-f sensorimotor coordination; $\mathbf{g}$ exploratory activity; $\mathbf{h}-\mathbf{j}$ anxiety behaviors; and $\mathbf{k}$ autogrooming. Only H-DE-71 F1 showed decreased mean values in $\mathbf{a}, \mathbf{b}, \mathbf{g}, \mathbf{k}$ and increased $\mathbf{i}$ whereas F0 exposed to L-DE-71 showed increased mean value in $\mathbf{i} . * P<.05$, $* * P<.01$ compared to corresponding VEH/CON. ${ }^{\wedge} P<.05,{ }^{\wedge} \wedge P<.01$ compared to corresponding L-DE-71. $n$ for F1 (litters/group): a 10-11; b 10; c 9-11; d 10-11; e 11-12; f 10-12; g 10-12; h 10-14; i 7-8; j 9-11; k 10-12. $n$ for F0 (subjects/group): a 21-27; b 17-22; c 22-26; d 16-20; e 19-27; f 16-20; g 22-27; h 22-27; i 16-26; j 22-25; k 21-27

\section{Discussion}

Growing evidence suggests a positive association between early-life exposure to PBDEs and neurodevelopmental alterations (Branchi et al. 2003). Environmental factors, including xenobiotic chemical exposures, may provide a plausible explanation for the rising incidence of NDDs characterized by social deficits (Maenner et al. 2020); however, experimental evidence has not established a direct link with specific candidate chemicals. With this purpose, our study is the first to investigate the effects of the penta-PBDE mixture DE-71 on behaviors and neurochemical/endocrine profiles relevant to several core ASD symptom domains concurrently. Our experimental design exposes progeny to the full complement of congeners found in human breast milk (Lind et al. 2003). The major findings reveal that developmental DE-71 exposure produces durable deficits in social recognition, repetitive behavior and social odor discrimination in exposed female offspring. The behavioral phenotypes occurred concomitantly with changes in plasma AVP and neuromolecular markers of $O x t$ and Avp signaling pathways 
Fig. 7 Early-life PBDE exposure does not alter locomotion on the open field test. $\mathbf{a}, \mathbf{b}$ Distance traveled in the open field arena. All F1 exposure groups showed similar reduced exploratory activity and velocity over the 1 h. c, d Exploration time in periphery and center for all groups showed habituation only in the periphery. $\mathbf{e}$ Exploration time in center was significantly less than in periphery for all groups, suggesting no exposure effects on anxiety. f Another measure of anxiety, number of fecal boli, indicated increased emotional reactivity in the L-DE-71 F1 relative to VEH/CON. $* P<.05, * * P<.01$. $* * * * P<.0001$ compared to center (e) or VEH/CON (f). $\wedge P<.05$ compared to corresponding L-DE-71. ${ }^{\mathrm{a}} P<.0001$ compared to initial time bin for corresponding treatment group. $n=19-23$ subjects/group. $C$ center zone, $P$ periphery zone
Fig. 8 DE-71 exposure alters prosocial gene expression in select brain regions involved in social behavior in F1 females. Heatmap representation (double gradient, blue-minus; redplus) of RT-qPCR analysis with the respective fold-change value (mean) of each gene studied by brain region. $n=4-17 /$ group. $* P<.05$ compared to VEH/ CON. $\wedge^{\wedge} P<.05$ compared to L-DE-71. BNST, bed nucleus of the stria terminalis; $A M G$ amygdala, $L S$ lateral septum, $S O N$ supraoptic nucleus, $P V N$ paraventricular nucleus

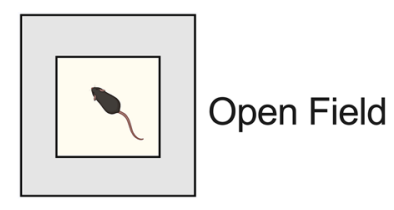

A

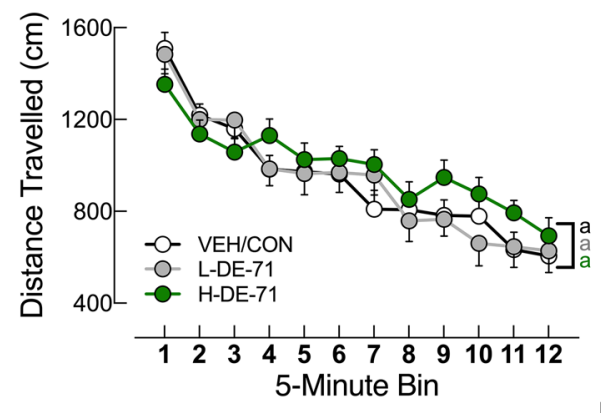

C

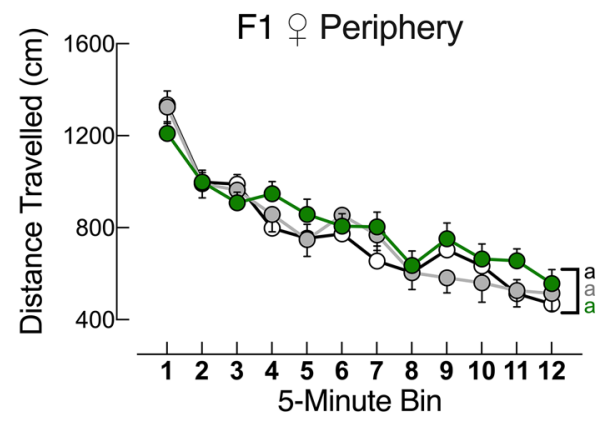

E

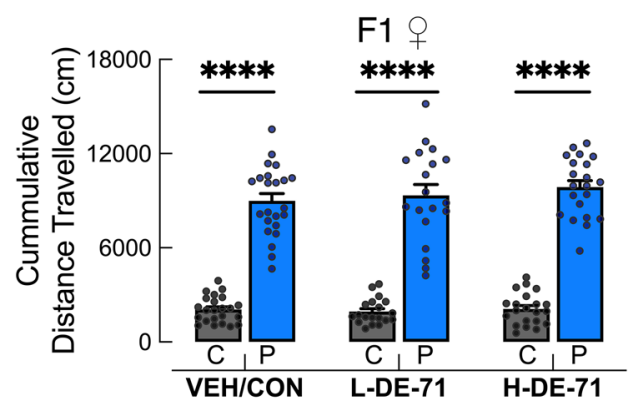

B

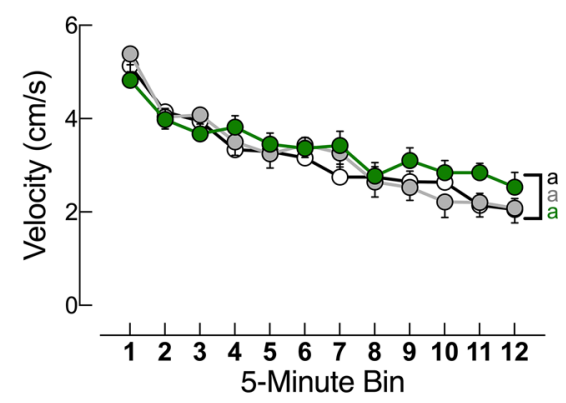

D

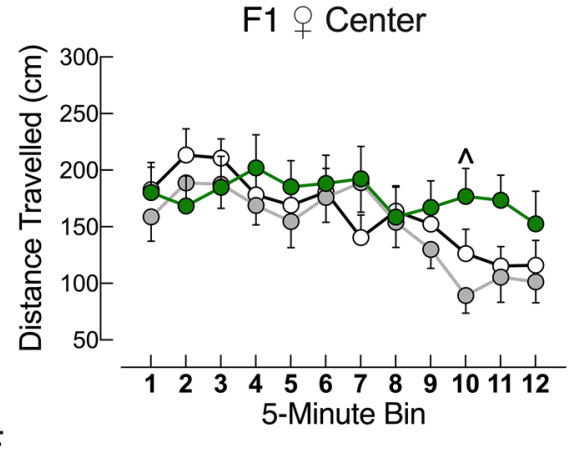

$\mathrm{F}$

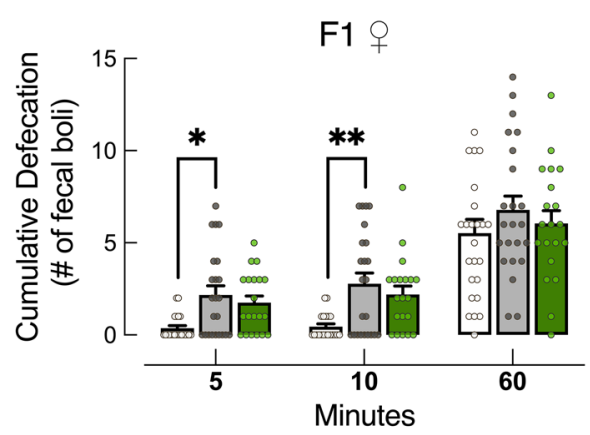

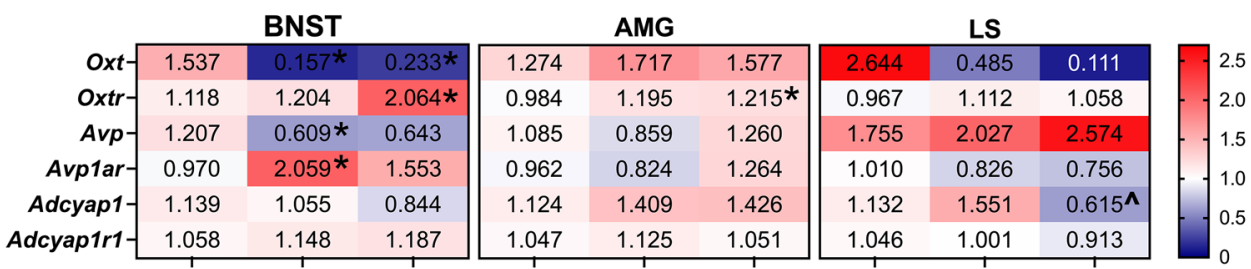

VEH/CON L-DE-71 H-DE-71 VEH/CON L-DE-71 H-DE-71 VEH/CON L-DE-71 H-DE-71

SON

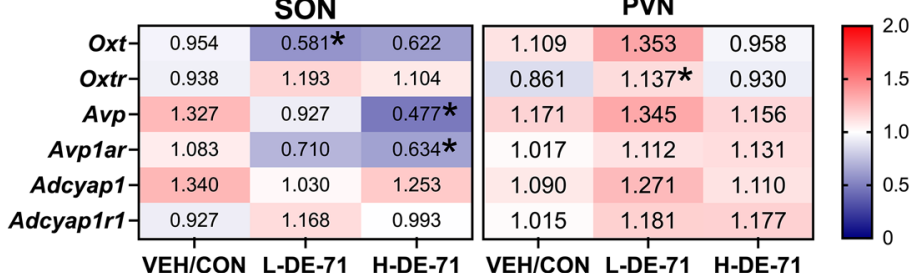

VEH/CON L-DE-71 H-DE-71 VEH/CON L-DE-71 H-DE-71 
Fig. 9 Perinatal exposure to DE-71 exaggerates plasma levels of Arg8-vasopressin but not oxytocin (OXT) in adult F1 female offspring. a Plasma Arg8-vasopressin measured using EIA using blood taken at sacrifice. L-DE-71 exposed offspring showed elevated levels. b OXT levels showed no exposure effects. $* P<.05$ compared to VEH/CON. $n=8-13$ subjects/ group (a); $n=6-8$ subjects/ group (b)

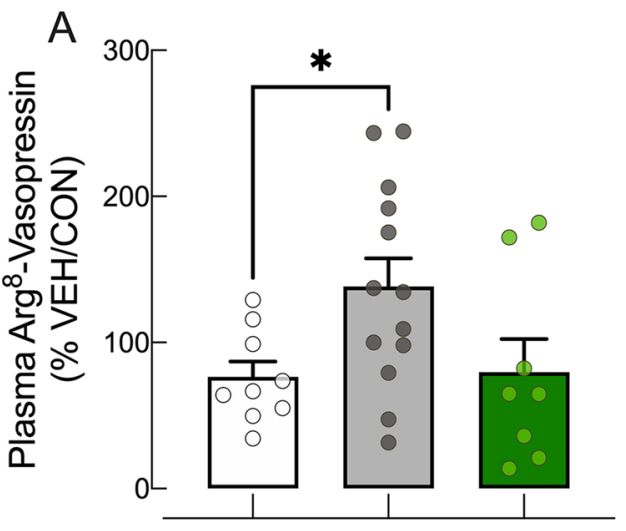

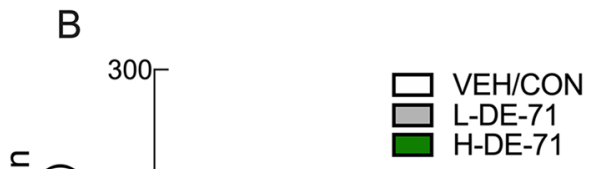
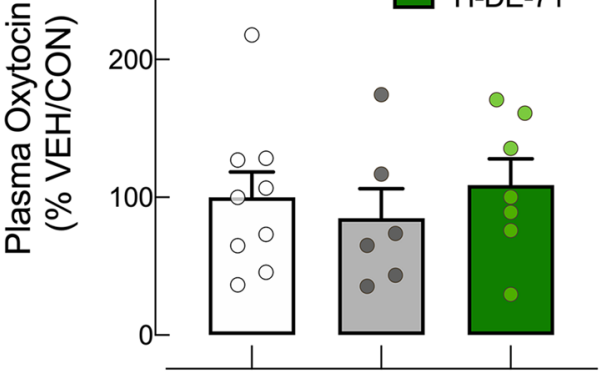

in brain regions that coordinate complex social behaviors. Together, the behavioral, sensory and neurochemical phenotypes produced by DE-71 may provide a novel, comprehensive ASD-relevant model with high translational impact. Our results are congruent with a disrupted developmental trajectory of the social processing domain as outlined in the 2010 NIMH Research Domain Criteria (RDoC) framework (Social Processes: Workshop Proceedings, 2012); a key characteristic of ASD pathology. Our work is further strengthened by the use of the litter as the unit of statistical analysis, thus minimizing risk of bias (RoB) of individual studies (Dorman et al. 2018) and inter-individual variability. Further, DE-71 produced the common hormetic response, such that the 0.1 but not $0.4 \mathrm{mg} / \mathrm{kg}$ group exhibited most of the behavior changes even though there was a dose-dependent increase in brain accumulation of $\sum$ PBDE congeners. Moreover, we confirmed the augmented susceptibility to developmental relative to adult exposure to PBDEs, highlighting the significance of chemical exposures during critical neurodevelopmental windows. Collectively, these data support the conclusion that environmental xenobiotics impact social behavior and related neurochemical signaling pathways in mice relevant to NDDs.

\section{Perinatal DE-71 exposure produces deficient social recognition and increases repetitive behavior in adult female offspring}

Our main finding was that in utero and lactational transfer of DE-71 from exposed mothers produces behavioral phenotypes resembling two core behavioral features of ASD DSM-V diagnosis: deficits in social reciprocity and communication and repetitive/stereotyped behaviors (American Psychiatric Association 2013). With respect to the latter, female offspring exposed to L-DE-71 showed increased activity on a marble burying test indicative of repetitive behaviors in rodent models of ASD (Angoa-Pérez et al. 2013). At $0.1 \mathrm{mg} / \mathrm{kg}$, developmental DE-71 exposure also produced deficient short-term social memory (SNP) and long-term social recognition memory (SRM), while sociability (SOC) was not affected, ruling out a lack of the "social motivation' component of social cognition as an underlying contributor. SRM is considered to be another distinct behavioral domain and important for the 'knowledge of self and others' component of social cognition (Bicks et al. 2020).

Though much is still unknown about the neural correlates of social behavior, the social motivation and social recognition domains have been shown to be independent of each other. For instance, deficits in SNP can occur without decrements in sociability in other models of deficient social behavior induced by high fat diet (Hayashi et al. 2020) or C-section delivery (Morais et al. 2020). The former can be restored by OXT administration. In other reports, restoring OXT content in the PVN with probiotic therapy (L. reuteri), in maternal high fat diet and valproic acid offspring, rescues SOC and SNP, but not other ASD endophenotypes (Sgritta et al. 2019); (Buffington et al. 2016). Taken together, these results suggest that different mechanisms and/or circuitry govern the various social behavior domains that can be selectively isolated by experimental contrast and susceptibility to early-life PBDEs. Specifically, perinatal DE-71 exposure significantly compromises the social recognition domain of social cognition, which is more relevant to ASD since the behaviors related to knowledge of self and others such as facial recognition, empathy and evaluation of emotion of others are disrupted in ASD patients (Bicks et al. 2020); (Bradshaw et al. 2011).

Our findings indicate deficient short-term social recognition and long-term social recognition memory in L-DE$71 \mathrm{~F} 1$, suggesting our results may be translational to ASD and other NDDs characterized by psycho-social deficiencies. While findings of epidemiological studies evaluating associations between PBDEs and social deficits/ASD are mixed (Vuong et al. 2018); (Braun et al. 2014); (Gibson et al. 2018), a higher risk of poor social competence has been found with increasing postnatal exposure to BDE-47 (4 yr old child serum; (Gascon et al. 2011). BDE-47 levels in cord blood have also been positively associated with poor 
social domain development in 24-month-old toddlers (Ding et al. 2015). Previous rodent studies examining the effects of environmental pollutants on social behavior have produced inconsistent results perhaps due to heterogeneity of brominated (BFR) flame retardants used, timing of exposure, sex and/or model organism used. Importantly, the only other study examining the effects of DE-71 (0.3 and $1.6 \mathrm{ppm})$ on social behavior supports our findings. Fernie and colleagues (2005) found fewer and less appropriate pair-bonding and courtship behaviors in exposed captive kestrels (Fernie et al. 2005). In contrast, female mice offspring exposed to BDE-47 perinatally via the mother showed reduced sociability relative to controls (Woods et al. 2012) but no effect of BDE-47 (at $0.03 \mathrm{mg} / \mathrm{kg}$ ) was detected on SNP unless administered to genetically altered mice lacking methyl-CPG binding protein 2 (Mecp2), a frontal cortical protein negatively associated with ASD (Nagarajan et al. 2006). Also in contrast to our findings, male CD-1 mice developmentally exposed to BDE-47 $(0.2 \mathrm{mg} / \mathrm{kg})$ display reduced time with conspecfics but show no effect on SNP relative to controls (Kim et al. 2015). Moreover, using rats perinatally exposed to high doses of BDE-47 $(50 \mathrm{mg} / \mathrm{kg}) \mathrm{Li}$ and others (2021) report normal preference for stranger over familiar conspecific and for social stimulus over empty corral but with reduced time spent in exploration (Li et al. 2021). Using low doses of BDE-209 (0.12 ng/mouse/day, s.c.), Chen and colleagues (2019) did not observe deficient sociability nor SNP in exposed male mice offspring (Chen et al. 2019). Therefore, it appears that PBDE effects on social behavior may be congener- and/or dose-specific. Our findings are supported by perinatal exposure studies using another flame retardant mixture Firemaster $550(6.6 \mathrm{mg} / \mathrm{kg} /$ day $)$ and its BFR and organophosphate components $(3.3 \mathrm{mg} / \mathrm{kg} / \mathrm{d})$, which produce deficits in social recognition after $24 \mathrm{~h}$ retention in a sexand exposure-specific manner in rats (Witchey et al. 2020). Perinatal exposure to Firemaster 550 also produces abnormal partner preference in female prairie voles $(1 \mathrm{mg} / \mathrm{kg}$; Gillera et al. 2020). Other studies have reported adverse effects of PCBs (A1221) on mate preference and social behavior (Reilly et al. 2015); (Hernandez Scudder et al. 2020).

\section{Perinatal DE-71 exposure produces deficient novel object recognition memory in dams and adult female offspring}

A complex interplay between forebrain regions is responsible for normal social recognition (Bicks et al. 2020); (Tanimizu et al. 2017); (Ferguson et al. 2000) including hippocampal circuits underlying social memory formation and amygdalar circuits that process social signals such as volatile odorant pheromones that trigger social and reproductive behaviors (Kogan et al. 2000); (Noack et al. 2010). DE-71-exposed socially deficient mice also showed abnormal NOR memory suggesting abnormal function in hippocampus since the latter serves as an integration hub underlying both social and recognition memory (Hernandez et al. 2008, Pfaffl 2001, Pinson et al. 2016). Toxicological studies of developmentally administered single BDE congeners or DE-71 have not examined effects on NOR or SRM (Dorman et al. 2018). However, previous studies using peri/postnatally administered single BDE congeners such as BDE-153 $(0.9 \mathrm{mg} / \mathrm{kg} \mathrm{bw})$ and $-47(0.03 \mathrm{mg} / \mathrm{kg} \mathrm{bw})$ have shown neurotoxic actions on hippocampal-dependent function related to spatial memory (Koenig et al. 2012); (Dorman et al. 2018); (Viberg et al. 2003). In support of our findings, evidence from human studies suggest that more than one environmental BDE congener may produce risk for cognitive impairments in children. For example, several PBDEs found in maternal samples (BDE-47, 99, 100, 153) are associated with children's lowered IQ and cognitive scores (Herbstman et al. 2010); (Lam et al. 2017); (Azar et al. 2021), mental/ physical development (Eskenazi et al. 2013) and fine motor skills, attention and cognition (Chen et al. 2014).

It is not surprising that L-DE-71 F1 mice showed coincident deficient NOR memory and SRM. However, while deficient SRM was seen at both short and long-term retention times, NOR memory deficits were evident with short-term retention time only. Moreover, F0 showed deficits only in short-term NOR memory indicating that short-term social recognition ability and short-term novel object recognition memory are distinct constructs. Therefore, PBDEs may target different brain circuits participating in general and social memory processes and/or different neurochemical systems within each circuit. For example, hippocampal OXTRs are necessary for short-term social recognition but not novel object recognition memory in male mice (Raam et al. 2017).

\section{Perinatal exposure to DE-71 alters social odor discrimination in adult female offspring}

Recognition of conspecifics in rodents depends on proper identification, discrimination and processing of olfactory cues present in urine and secretions from skin, reproductive tract and scent glands (Kogan et al. 2000); (Noack et al. 2010). We found that the disruption of social behavior after perinatal DE-71 exposure is coincident with abnormal profiles of olfactory habituation/dishabituation to social odors. For example, socially deficient L-DE-71 mice also displayed a reduction in habituation to social odors and in dishabituation from one to another social odor. In H-DE-71 mice the deficits in olfactory discrimination were relatively less severe as was that of their social behavior (reduced sociability with normal social recognition ability and memory). In combination, these results suggest that DE-71 effects on olfactory discrimination are specific to social odors, since 
exposure produced no deficits in general olfactory processing, and that deficiency was coincident with social deficits in a dose-dependent manner. It is unclear why PBDEs are more neurotoxic to social odor processing but it may depend on differential targeting of CNS pathways taken by signals from neutral and social odors. Chemosensory cues are processed through two olfactory systems; neutral odors (banana and almond) are processed through the main olfactory epithelium (MOE) and social odors through both MOE and the vomeronasal organ (VNO) (Huckins et al. 2013). Signals are then processed through amygdala and hypothalamus to trigger innate social and reproductive behaviors.

There are no previous studies on PBDEs and olfactory function although BDE-47, -85, -99 can concentrate in the epithelium of the nasal cavity (Darnerud and Risberg 2006) and developmental exposure to BDE-209 impairs subventricular zone (SVZ) neurogenesis and olfactory granule cell morphology in mice (Xu et al. 2018). However, a recent study using PCBs (Aroclor 1221, $1 \mathrm{mg} / \mathrm{kg}$ ) indicates that prenatal exposure impairs mate preference behavior based on olfactory cues concomitant with impaired odor preference for mates with different hormone status in adult female offspring (Hernandez Scudder et al. 2020). Our findings that early-life PBDE exposure alters social odor discrimination may translate to autistic humans which are prone to hypo- or hyper-reactivity to sensory stimulation (American Psychiatric Association 2013); recent studies suggest that this may include atypical olfaction (Martin and Daniel 2014). Indeed, several olfactory outcomes have been reported in children with ASD, i.e., abnormal odor responses, difficulties in emotional reaction to odors, impaired detection thresholds and odor identification as well as heightened olfactory sensitivity (Martin and Daniel 2014); (Rogers et al. 2003); (Legiša et al. 2013). Further research is needed to discern the mechanisms by which PBDEs may act to alter social odor discrimination and if this underlies, in part, their social recognition deficits. Interestingly, extrahippocampal OXT and AVP systems that contribute to short-term social recognition, also modulate detection and processing of social odors (Wacker and Ludwig 2012); (Oettl et al. 2016).

\section{Perinatal DE-71 Alters AVP and OXTergic neuromolecular phenotypes in brain regions that coordinate complex social behaviors}

Our lab has previously shown that in vitro and early-life exposure to PBDEs (and PCBs) produce neuroendocrine disruption of the prosocial neuropeptide, vasopressin, under physiologically stimulated conditions (Coburn et al. 2005); (Coburn et al. 2007); (Mucio-Ramírez et al. 2017). Therefore, the observed PBDE-induced deficits in SNP and SRM may result from altered prosocial functions of AVP and/or the structurally related neuropeptide, OXT. Here we show that L-DE-71 downregulates Avp in BNST, which provides sexually dimorphic AVPergic innervation to LS (Bychowski et al. 2013). Diminished AVPergic signaling to LS may explain reduced social recognition memory in L-DE-71 F1 females, since local AVP1a receptor antagonism compromises social discrimination especially well in females (Veenema et al. 2012). DE-71-mediated upregulation in BNST Avplar may represent a compensatory effect to maintain signaling at normal levels. Interestingly, Avplar in the ventromedial nucleus (VMN) is upregulated by the PCB mixture A1221 in female rat (but not male) offspring and is not dependent on estrogenic pathways (Topper et al. 2019). The observed downregulation of Avp in SON may also impact social recognition ability indirectly via reduced AVPergic-mediated activation of BNST (Lukas et al. 2011).

At $0.1 \mathrm{mg} / \mathrm{kg}$, DE-71 also produced elevated plasma AVP which is consistent with less inhibitory regulation over axonal secretion of AVP hormone resulting from potentially reduced levels of central AVP (Ludwig et al. 2016). Additionally, DE-71 can increase exocytosis in PC12 pheochromocytoma endocrine cells (Dingemans et al. 2008) possibly by interfering with intracellular calcium dynamics (Coburn et al. 2008) and potentially increasing secretion of stored AVP depots in axonal terminals located in the posterior pituitary and, thus, releasing AVP into the bloodstream. We also found that DE-71 alters the central OXTergic system which is also necessary for social recognition and partner preference (Ferguson et al. 2000). For example, mice with OXT gene deletion fail to remember recently encountered individuals and do not show the typical decline in preference during subsequent exposures to a familiar mouse, an effect which can be rescued by central administration of OXT (Dluzen et al. 1998). Here we show that in the BNST, L-DE-71 female F1 display significantly reduced $O x t$ mRNA transcripts. Assuming that there is a positive correlation between gene and peptide content and release, one interpretation of our data is that L-DE-71 exposure reduces central OXTergic signaling which is necessary for normal social discrimination (Dumais et al. 2016). Our results further indicate that BNST-originating OXT may be sufficiently important for activating BNST OXTR relative to PVN-originating OXT (Knobloch et al. 2012). Indeed, a recent report has demonstrated that OXT receptor blockade, in the extrahypothalamic population of oxytocinergic neurons of the BNST, impairs social recognition in female and male rats (Dumais et al. 2016). OXT in the BNST also drives stress-induced social vigilance and avoidance that may be at play in social behavior domains examined here ${ }^{137}$. Ultimately, OXTergic signaling in the BNST may not be sufficient to affect social recognition since H-DE-71 F1 females also displayed reduced BNST $O x t$ mRNA. Importantly, L-DE-71 also reduced $O x t$ transcripts in the SON. Because local release of OXT from SON dendrites that extend to MeA promotes social recognition through amygdalar OXTRs (Takayanagi 
et al. 2017); (Abramova et al. 2020), downregulated $O x t$ in SON may underlie, in part, the associated SNP and SRM deficits. Since the promoter regions for genes of both oxytocin (Mamrut et al. 2013) and vasopressin systems (Auger et al. 2011) are susceptible to epigenetic modification (Auger et al. 2011), these genes may be altered by global DNA methylation that is possible after developmental BDE-47 exposure (Woods et al. 2012); (Poston and Saha 2019). Our findings may have translational value since altered OXT and AVP mechanisms in humans have been implicated in ASD and other psychiatric and neurological disorders (Zhang et al. 2016); (Kobylinska et al. 2019); (Hendaus et al. 2019); (Oztan et al. 2018).

Growing evidence from a myriad of studies using different model organisms show that brain vasopressin and oxytocin systems may be targets of other EDCs. EDC chemicals include phytoestrogens like genestein, chlorpyrifos, diethystilbestrol, bisphenol A (BPA), organophosphate pesticides and organohalogens like PCBs, PBDEs and Firemaster 500 (Patisaul 2017). For example, we and others have shown that some of these EDCs such as Firemaster 550 (Gillera et al. 2021), PCBs such as A122 (Reilly et al. 2021) and A1254 (Coburn et al. 2005); (Coburn et al. 2007) and BPA (Witchey et al. 2020) have endocrine disruptive effects on the oxytocin and/or vasopressin systems and, in some cases, these are concomitant with their effects on social behavior (Witchey et al. 2020); (Gillera et al. 2020); (Hernandez Scudder et al. 2020); (Reilly et al. 2021).

\section{Specificity and comprehensive profile of PBDE toxicant model of ASD}

A recent meta-review has put forth recommendations to improve ASD model characterization in rodent studies such that information about reciprocal social communication and stereotyped repetitive behavior domains are characterized in the same animals (Pelch et al. 2019). To this end, we used established protocols to measure ASD relevant and other comorbid behaviors to fully characterize the DE-71-induced phenotypes (Moy et al. 2004); (Crawley 2012). We found that the effects of DE-71 were specific to social novelty preference and social recognition memory as well as repetitive behavior and olfactory discrimination of social odors. Alterations were specific to offspring exposed perinatally via maternal transfer of environmentally relevant BDE congeners; adult exposed mothers were mostly unaffected. DE-71 had little to no effects on behaviors representing the domains of anxiety, depression and locomotion indicating ASD-relevant specificity without general neurological effects. In addition, there were no indications of reduced general health, i.e., body weight in pups nor gross abnormalities in maternal nest conditions. We have recently reported that similarly exposed (L-DE-71) female offspring, and to a lesser degree, their exposed mothers, display diabetic symptomatology, effects which may relate to the present findings (Kozlova et al. 2020). Importantly, we used multiple behavioral tests to validate social and other constructs studied (locomotion and anxiety). For example, for all F1 groups, the frequency of total entries on EPM and distance travelled on OFT yielded similar results regarding locomotion. In addition, time spent in open arm on EPM, and latency to leave center on Suok was consistent with duration in center of OFT.

Our DE-71 model of ASD also shows altered prosocial peptide neurotransmitters/neurohormones that are critical to ensuring proper development of social brain networks. In particular, the vasopressin and oxytocin systems are critically involved in social cognition with mutations having socio-behavioral impact that have been implicated in core symptoms of autism (Frank and Landgraf 2008). These neurochemical systems are being actively studied as potential targets of future therapeutic interventions for ASD (MeyerLindenberg et al. 2011); (Bolognani et al. 2019). In light of incongruent findings reported by past rodent and human studies (Pelch et al. 2019), we believe that our findings brings us closer to understanding the risk of ASD posed by xenobiotic endocrine disrupting chemicals. Nevertheless, human and rodent studies reporting on the relationship between PBDE exposure and autistic phenotype are few in number and have yielded inconclusive results and this field would benefit from additional detailed epidemiological and animal studies on the relationship between persistent organic pollutants (POPs) and risk of ASD.

\section{Maternal transfer of BDE congeners in DE-71 and their brain accumulation in female offspring is dose- and time-dependent}

BDE congener composition found in PND15 exposed brains mimics that found in humans. BDE-28, $-47,-99,-100,-153$ were common congeners found at ppb in both DE-71-exposed offspring groups at PND15 with three-fold greater levels in H-DE-71 than L-DE-71. $\sum$ PBDE values for adult serum are 30-100 ng/g lipid (Costa and Giordano 2007) and 3 to ninefold higher in infants because of exposure through breastmilk and in toddlers because of exposure through house dust and the diet (Toms et al. 2008); (Rose et al. 2010); (Schecter et al. 2005); (Fischer et al. 2006). Serum $\sum$ PBDE values can reach $482 \mathrm{ng} / \mathrm{g}$ l.w. in toddlers (California 18 month-old) (Fischer et al. 2006) but lesser values have also been reported, i.e., $127 \mathrm{ng} / \mathrm{g}$ 1.w. (Vuong et al. 2017) in Ohio 2-year-olds and $100 \mathrm{ng} / \mathrm{g}$ l.w. for North Carolina 12-36 month-old toddlers (Stapleton et al. 2012). Using a divisor factor of 0.095 to convert w.w. to 1.w. (unpublished observations), we estimate our mean $\sum$ PBDE in L-DE-71 F1 at PND 15 to be 1.7- to 8.2-fold greater, suggesting ours represents a translational model of maternal PBDE transfer. The main congeners in PND15 brains, BDE-47, -85, 
$-99,-100,-153$, and -154 , accounting for $97 \%$ of the mean $\sum$ PBDEs, also comprise the majority of congeners (96\%) in DE-71 (Kodavanti et al. 2010). These and other congeners found in offspring brain samples, i.e., BDE-17, 28, 49, 138, $139,140,183$ and 184, have also been detected in human serum and/or breastmilk (Chao et al. 2010). Importantly, to our knowledge, BDE-49, -140, -183, -184 have not been previously detected in DE-71 exposed rodent brain (Kodavanti et al. 2010). Kodavanti et al. 2010 have reported brain levels of PBDEs in adult offspring after exposing dams during pregnancy and lactation to high levels of DE-71 (30 mg/ $\mathrm{kg}$ ) (Kodavanti et al. 2010). Other papers have reported brain BDE levels after maternal transfer or directly exposing postnatal or adult mice with BDE congeners (Darnerud and Risberg 2006); (Staskal et al. 2006). Our study is unique because we used environmentally relevant congeners and doses $(0.1$ and $0.4 \mathrm{mg} / \mathrm{kg})$ in a maternal transfer model with translational value. We also examined penetration of congener in exposed offspring during both early postnatal development and as adults.

Although using a mixture like DE-71 closely models the PBDE contamination previously shown in human breastmilk, there are some congeners, found at low levels in breastmilk, that we did not detect in offspring brain, i.e., BDE-7, 15, 71, 77, 119, 126 (Zhao et al. 2013); (Chao et al. 2010). Of these BDE-71 and -126 are present in DE-71 (LaA Guardia et al. 2006). Little or no information is available about the penetrance and/or neuroactivity of the missing congeners. Most rodent studies, which have focused on a single PBDE congener, BDE-47, dominantly detected in humans, have not reported pervasive effects on social behavior as we do here using DE-71. We speculate that BDE-47 alone is not effective in producing deficits in social recognition ability and social memory and that, instead, several PBDE congeners may act synergistically and/or additively to generate these abnormal phenotypes, reinforcing the need for in vivo studies using PBDE formulations that mimic child exposure.

By PND 110, the BDE composition in F1 brain was limited to BDE-153. Minimal metabolism of this congener is observed in rodents due to its high lipophilicity as determined by a high octanol-water partition coefficient (Log $\mathrm{K}_{\mathrm{ow}}$ ) (Sanders et al. 2006). BDE-153 has been positively associated with lower IQ in children and can cause impaired learning and memory in animal studies (Viberg et al. 2003); (Azar et al. 2021). However, while BDE-153 (and an additional 6 congeners) are detected at ppb in postmortem brain samples from 4-71 year-old born 1940 to 2000, it seems to be significantly depleted in autistics relative to normal subjects (Mitchell et al. 2012) for unknown reasons although this findings is based on a small sample size. The relatively lower retention of BDE-47 is in line with a previous report of differential tissue accumulation and disposition of
BDE congeners attributed to their toxicokinetic properties (Staskal et al. 2006). Cyp-mediated biotransformation of BDE-47 and -99 (but not 153) may contribute since these congeners contain sites with adjacent unsubstituted carbons where the metabolism occurs (Sanders et al. 2006). Our findings suggest that elevated brain levels of BDE congeners in DE-71 during the critical window of early postnatal neurodevelopment may predispose children to neurobehavioral alterations related to ASD.

\section{Conclusion}

Though the role of environmental toxicants in the etiology of NDDs is poorly understood, our data support a link between maternal toxicant exposures and abnormal social and repetitive behavior in offspring that is relevant to ASD. We have shown that early-life exposure to DE-71 leading to these phenotypes is associated with humanrelevant levels and composition of BDE congeners penetrating the postnatal offspring brain via maternal transfer. DE-71 exposure has prominent actions if it occurs during perinatal development as compared to adulthood, supporting previous studies showing the particular susceptibility of developing nervous system to neurotoxic actions of PBDEs. The abnormal social behavior phenotypes produced by DE-71 are mostly specific to social novelty preference and social recognition memory and are also associated with excessive repetitive behavior, as well as neurochemical and social odor processing correlates-suggesting that discrete brain functional systems are targeted by PBDEs to promote neurodevelopmental abnormalities. Future studies are needed to discern if DE-71 actions are sexually dimorphic and extend to exposed male offspring. We believe that our environmental toxicant mouse model has utility in future studies examining the relationship between environmental xenobiotics, neurodevelopmental reprogramming and the rising incidence of NDDs.

\section{Limitations of the study}

The results of the PCR analysis provide novel results on the effects of PBDE exposure on the expression of gene markers for 'prosocial' neuropeptides and their receptors in specific regions of the social brain network. However, these restricted regions vary in cell density and limit the RNA yield for genes of interest (GOIs), especially in the amygdala and LS. Moreover, relative expression was more variable for ROIs that have low expression of GOIs, i.e., Oxt for LS. To improve our experimental data, we followed MIQE guidelines to optimize oligonucleotide primer efficiency and target specificity. Nevertheless, since 
the methodological approach we outlined depends on the level and variability of gene expression and quantity of RNA collected, our results should be interpreted alongside these limitations. BDE congener analysis was performed using two mass spectrometry methods utilized by teams at different institutions. The GC/ECNI-MS method uses an ECNI ionization mode to improve sensitivity. This method provides equal sensitivity to that provided by HRGC/ HRMS that uses electron impact ionization. Therefore, the reduction in brain BDE congeners at PND110 is likely due to elimination after accumulation measured at PND 15 and not to methodological factors. The great majority of behavioral tests were analyzed using litter as the unit of statistical analysis. However, for practical reasons most other tests used individual subjects. Our findings pertain to exposed female offspring and their mothers but male offspring were omitted due to limited resources. Further research is needed to determine if the ASD phenotypes evoked using the PBDE model are sex-specific.

Supplementary Information The online version contains supplementary material available at https://doi.org/10.1007/s00204-021-03163-4.

Acknowledgements We acknowledge Drs. G. Hicks, M. Collin, D. Carter and C. and H. Clark at UCR Institute for Integrative Genome Biology, Genomics and Imaging Cores, E. Grace (IDT) and M. Kuhn (NEB) for troubleshooting advice on PCR. We thank UCR graduate students R. Bottom, K. Conner and D. Rohac for assistance with Suok and FST. We thank Drs. K. Huffman (Dept. Psychology), W. Saltzman (Dept. Biology) for access to their behavioral apparati. We are grateful to Dr. B. Wong (Noldus) for Ethovision software training. Dr. J. Porter and M. Colon at the Brain Behavioral Core (RR003050/MD007579), Ponce Health Sciences University, provided additional support on behavioral data analysis. We thank B. O'Hara and R. Hart (Arbor Assays) for advice on sample preparation for peptide EIA and GraphPad Support staff for helpful software assistance. We thank J. Phan for help with animal husbandry, Drs. S. Gill and S. Haga-Yamanaka for use of plate readers and Dr. M. Adams for the use of speed vacuum evaporator. We are grateful to Drs. I. Ethell, F. Sladek, K. Huffman, M. Riccomagno for gift of mice used as breeders and stimulus animals. Illustrations were created with BioRender.com. The authors also thank Drs. Michael Hughes and Andrew Johnstone of USEPA for their helpful comments on an earlier version of this manuscript.

CRediT authorship contribution statement Elena V. Kozlova: conceptualization, data curation, formal analysis, funding acquisition, investigation, methodology, project administration, supervision, validation, visualization, writing — original draft, writing-review and editing. Matthew C. Valdez: conceptualization, data curation, formal analysis, funding acquisition, investigation, methodology, project administration, supervision, validation. Maximilian E. Denys: formal analysis, funding acquisition, investigation, writing — original draft. Anthony E. Bishay: formal analysis, funding acquisition, investigation, writing-original draft. Julia M. Krum: data curation, funding acquisition, investigation, methodology, visualization. Kayhon M. Rabbani: formal analysis, funding acquisition, investigation, validation, data curation. Valeria Carrillo: investigation, funding acquisition, data curation. Gwen M. Gonzalez: funding acquisition, investigation, methodology, validation. Jasmin D. Tran: formal analysis, investigation, funding acquisition. Brigitte M. Vazquez: investigation, funding acquisition. Gregory Lampel: investigation, funding acquisition. Laura M. Anchondo: investigation. Syed A. Uddin: investigation, validation. Nicole M. Huffman: investigation, validation. Eduardo Monarrez: investigation, data curation, validation. Duraan S. Olomi: investigation, data curation. Bhuvaneswari D. Chinthirla: investigation. Richard E. Hartman: resources, methodology, validation, writing - review and editing. Prasada Rao S. Kodavanti: funding acquisition, resources, writing - review and editing. Gladys Chompre: investigation. Allison L. Phillips: formal analysis, investigation, writing - review and editing. Heather M. Stapleton: formal analysis, funding acquisition, methodology, resources, supervision, validation, writing — review and editing. Bernhard Henkelmann: investigation, methodology, validation, writing — original draft. Karl-Werner Schramm: methodology, resources, funding acquisition, supervision, writing - review and editing. Margarita C. Curras-Collazo: conceptualization, data curation, formal analysis, funding acquisition, investigation, methodology, project administration, resources, supervision, validation, visualization, writing — original draft, writing — review and editing.

Funding We acknowledge funding from UCR Committee on Research (CoR) Grants to M.C.C.; UC MEXUS Awards to M.C.C., E.V.K., M.C.V.; NSF GRFP to M.C.V.; MARC U STAR Fellowship and NIH T34 (T34GM062756) to G.M.G.; UCR GRMP to E.V.K.; Sigma Xi Grant-in-Aid of Research award to E.V.K., K.M.R., M.E.D.; UCR Undergraduate Minigrant to E.V.K., K.M.R., A.E.B., V.C., G.L., B.M.V.; STEM-HSI Department of Education Award to E.V.K.; UCR Chancellor's Fellowship to J.M.K., APS IOSP Scholarship to L.M.A, APS STRIDE to A.E.B., and NIH R01 ES016099 to H.M.S.

Availability of data and materials Not applicable.

Code availability Not applicable.

\section{Declarations}

Conflict of interests The authors report no conflicts of interests and have no competing interests to declare.

Disclaimer J.M.K. is now a second Lieutenant at the Uniformed Services University, Department of Defense. Her work was performed at the University of California, Riverside before becoming a military officer. However, we want to emphasize that the opinions and assertions expressed herein are those of the authors and do not necessarily reflect the official policy or position of the Uniformed Services University or the Department of Defense. The research described in this article has been reviewed by the Center for Public Health and Environmental Assessment, U.S. Environmental Protection Agency (EPA) and approved for publication. Approval does not signify that the contents necessarily reflect the views and policies of the agency nor does the mention of trade names of commercial products constitute endorsement or recommendation for use.

Ethics approval Care and treatment of animals was performed in accordance with guidelines from and approved by the University of California, Riverside Institutional Animal Care and Use Committee (AUP \#20170026 and 20200018).

Consent to participate Not applicable.

Consent for publication All authors reviewed and approved the final manuscript.

Open Access This article is licensed under a Creative Commons Attribution 4.0 International License, which permits use, sharing, 
adaptation, distribution and reproduction in any medium or format, as long as you give appropriate credit to the original author(s) and the source, provide a link to the Creative Commons licence, and indicate if changes were made. The images or other third party material in this article are included in the article's Creative Commons licence, unless indicated otherwise in a credit line to the material. If material is not included in the article's Creative Commons licence and your intended use is not permitted by statutory regulation or exceeds the permitted use, you will need to obtain permission directly from the copyright holder. To view a copy of this licence, visit http://creativecommons. org/licenses/by/4.0/.

\section{References}

Abbasi G, Li L, Breivik K (2019) Global Historical Stocks and Emissions of PBDEs. Environ Sci Technol 53:6330-6340. https://doi. org/10.1021/acs.est.8b07032

Abramova O, Zorkina Y, Ushakova V et al (2020) The role of oxytocin and vasopressin dysfunction in cognitive impairment and mental disorders. Neuropeptides 83:102079. https://doi.org/10.1016/j. npep.2020.102079

Abu-Arafeh A, Jordan H, Drummond G (2016) Reporting of method comparison studies: a review of advice, an assessment of current practice, and specific suggestions for future reports. $\mathrm{Br}$ J Anaesth 117:569-575. https://doi.org/10.1093/bja/aew320

Adgent MA, Hoffman K, Goldman BD et al (2014) Brominated flame retardants in breast milk and behavioural and cognitive development at 36 months. Paediatr Perinat Epidemiol 28:48-57. https://doi.org/10.1111/ppe.12078

American Psychiatric Association (2013) Diagnostic and Statistical Manual of Mental Disorders (DSM-5®). American Psychiatric Pub

Alvarez-Gonzalez MY, Sánchez-Islas E, Mucio-Ramirez S et al (2020) Perinatal exposure to octabromodiphenyl ether mixture, DE-79, alters the vasopressinergic system in adult rats. Toxicol Appl Pharmacol 391:114914. https://doi.org/10.1016/j. taap.2020.114914

Angoa-Pérez M, Kane MJ, Briggs DI et al (2013) Marble burying and nestlet shredding as tests of repetitive, compulsive-like behaviors in mice. J vis Exp. https://doi.org/10.3791/50978

Auger CJ, Coss D, Auger AP, Forbes-Lorman RM (2011) Epigenetic control of vasopressin expression is maintained by steroid hormones in the adult male rat brain. Proc Natl Acad Sci U S A 108:4242-4247. https://doi.org/10.1073/pnas.1100314108

Azar N, Booij L, Muckle G et al (2021) Prenatal exposure to polybrominated diphenyl ethers (PBDEs) and cognitive ability in early childhood. Environ Int 146:106296. https://doi.org/10. 1016/j.envint.2020.106296

Bicks LK, Yamamuro K, Flanigan ME et al (2020) Prefrontal parvalbumin interneurons require juvenile social experience to establish adult social behavior. Nat Commun 11:1003. https:// doi.org/10.1038/s41467-020-14740-z

Bielsky IF, Hu S-B, Szegda KL et al (2004) Profound impairment in social recognition and reduction in anxiety-like behavior in vasopressin V1a receptor knockout mice. Neuropsychopharmacology 29:483-493. https://doi.org/10.1038/sj.npp.1300360

Bolognani F, Del Valle RM, Squassante L et al (2019) A phase 2 clinical trial of a vasopressin V1a receptor antagonist shows improved adaptive behaviors in men with autism spectrum disorder. Sci Transl Med. https://doi.org/10.1126/scitranslm ed.aat7838

Bradshaw J, Shic F, Chawarska K (2011) Brief report: face-specific recognition deficits in young children with autism spectrum disorders. J Autism Dev Disord 41:1429-1435. https://doi.org/ 10.1007/s10803-010-1150-4

Branchi I, Alleva E, Costa LG (2002) Effects of perinatal exposure to a polybrominated diphenyl ether (PBDE 99) on mouse neurobehavioural development. Neurotoxicology 23:375-384. https:// doi.org/10.1016/s0161-813x(02)00078-5

Branchi I, Capone F, Alleva E, Costa LG (2003) Polybrominated diphenyl ethers: neurobehavioral effects following developmental exposure. Neurotoxicology 24:449-462. https://doi.org/10.1016/ S0161-813X(03)00020-2

Braun JM, Kalkbrenner AE, Just AC et al (2014) Gestational exposure to endocrine-disrupting chemicals and reciprocal social, repetitive, and stereotypic behaviors in 4- and 5-year-old children: the HOME study. Environ Health Perspect 122:513-520. https://doi. org/10.1289/ehp.1307261

Brennan PA, Kendrick KM (2006) Mammalian social odours: attraction and individual recognition. Philos Trans R Soc Lond B Biol Sci 361:2061-2078. https://doi.org/10.1098/rstb.2006.1931

Buffington SA, Di Prisco GV, Auchtung TA et al (2016) Microbial reconstitution reverses maternal diet-induced social and synaptic deficits in offspring. Cell 165:1762-1775. https://doi.org/10. 1016/j.cell.2016.06.001

Bustin SA, Benes V, Garson JA et al (2009) The MIQE guidelines: minimum information for publication of quantitative real-time PCR experiments. Clin Chem 55:611-622. https://doi.org/10. 1373/clinchem.2008.112797

Bychowski ME, Mena JD, Auger CJ (2013) Vasopressin infusion into the lateral septum of adult male rats rescues progesteroneinduced impairment in social recognition. Neuroscience 246:5258. https://doi.org/10.1016/j.neuroscience.2013.04.047

Chao HA, Chen SC-C, Chang C-M et al (2010) Concentrations of polybrominated diphenyl ethers in breast milk correlated to maternal age, education level, and occupational exposure. J Hazard Mater 175:492-500. https://doi.org/10.1016/j.jhazmat.2009.10.032

Chao H-R, Tsou T-C, Huang H-L, Chang-Chien G-P (2011) Levels of breast milk PBDEs from southern Taiwan and their potential impact on neurodevelopment. Pediatr Res 70:596-600. https:// doi.org/10.1203/PDR.0b013e3182320b9b

Chen Z-J, Liu H-Y, Cheng Z et al (2014) Polybrominated diphenyl ethers (PBDEs) in human samples of mother-newborn pairs in South China and their placental transfer characteristics. Environ Int 73:77-84. https://doi.org/10.1016/j.envint.2014.07.002

Chen Y, Liu S, Xu H et al (2019) Maternal exposure to low dose BDE209 and $\mathrm{Pb}$ mixture induced neurobehavioral anomalies in C57BL/6 male offspring. Toxicology 418:70-80. https://doi.org/ 10.1016/j.tox.2019.02.016

Coburn CG, Gillard ER, Currás-Collazo MC (2005) Dietary exposure to aroclor 1254 alters central and peripheral vasopressin release in response to dehydration in the rat. Toxicol Sci 84:149-156. https://doi.org/10.1093/toxsci/kfi046

Coburn CG, Currás-Collazo MC, Kodavanti PRS (2007) Polybrominated diphenyl ethers and ortho-substituted polychlorinated biphenyls as neuroendocrine disruptors of vasopressin release: effects during physiological activation in vitro and structureactivity relationships. Toxicol Sci 98:178-186. https://doi.org/ 10.1093/toxsci/kfm086

Coburn CG, Currás-Collazo MC, Kodavanti PRS (2008) In vitro effects of environmentally relevant polybrominated diphenyl ether (PBDE) congeners on calcium buffering mechanisms in rat brain. Neurochem Res 33:355-364. https://doi.org/10.1007/ s11064-007-9430-x

Coburn CG, Watson-Siriboe A, Hou B et al (2015) Permanently compromised NADPH-diaphorase activity within the osmotically activated supraoptic nucleus after in utero but not adult exposure to Aroclor 1254. Neurotoxicology 47:37-46. https://doi.org/10. 1016/j.neuro.2014.12.009 
Costa LG, Giordano G (2007) Developmental neurotoxicity of polybrominated diphenyl ether (PBDE) flame retardants. Neurotoxicology 28:1047-1067. https://doi.org/10.1016/j.neuro.2007.08.007

Costa LG, de Laat R, Tagliaferri S, Pellacani C (2014) A mechanistic view of polybrominated diphenyl ether (PBDE) developmental neurotoxicity. Toxicol Lett 230:282-294. https://doi.org/10. 1016/j.toxlet.2013.11.011

Crawley JN (2012) Translational animal models of autism and neurodevelopmental disorders. Dialogues Clin Neurosci 14:293-305

Currás-Collazo MC (2011) Nitric oxide signaling as a common target of organohalogens and other neuroendocrine disruptors. J Toxicol Environ Health B Crit Rev 14:495-536. https://doi.org/10. 1080/10937404.2011.578564

Darnerud PO (2008) Brominated flame retardants as possible endocrine disrupters. Int J Androl 31:152-160. https://doi.org/10.1111/j. 1365-2605.2008.00869.x

Darnerud PO, Risberg S (2006) Tissue localisation of tetra- and pentabromodiphenyl ether congeners (BDE-47, -85 and -99) in perinatal and adult C57BL mice. Chemosphere 62:485-493. https:// doi.org/10.1016/j.chemosphere.2005.04.004

Darnerud PO, Lignell S, Aune M et al (2015) Time trends of polybrominated diphenylether (PBDE) congeners in serum of Swedish mothers and comparisons to breast milk data. Environ Res 138:352-360. https://doi.org/10.1016/j.envres.2015.02.031

Darrow LA, Jacobson MH, Preston EV et al (2017) Predictors of serum polybrominated diphenyl ether (PBDE) concentrations among children aged 1-5 years. Environ Sci Technol 51:645-654. https://doi.org/10.1021/acs.est.6b04696

Deol P, Kozlova E, Valdez M et al (2020) Dysregulation of hypothalamic gene expression and the oxytocinergic system by soybean oil diets in male mice. Endocrinology. https://doi.org/10.1210/ endocr/bqz044

Ding G, Yu J, Cui C et al (2015) Association between prenatal exposure to polybrominated diphenyl ethers and young children's neurodevelopment in China. Environ Res 142:104-111. https://doi.org/ 10.1016/j.envres.2015.06.008

Dingemans MML, de Groot A, van Kleef RGDM et al (2008) Hydroxylation increases the neurotoxic potential of BDE-47 to affect exocytosis and calcium homeostasis in PC12 cells. Environ Health Perspect 116:637-643. https://doi.org/10.1289/ehp.11059

Dingemans MML, van den Berg M, Westerink RHS (2011) Neurotoxicity of brominated flame retardants: (in)direct effects of parent and hydroxylated polybrominated diphenyl ethers on the (developing) nervous system. Environ Health Perspect 119:900-907. https://doi.org/10.1289/ehp.1003035

Dluzen DE, Muraoka S, Engelmann M, Landgraf R (1998) The effects of infusion of arginine vasopressin, oxytocin, or their antagonists into the olfactory bulb upon social recognition responses in male rats. Peptides 19:999-1005. https://doi.org/10.1016/s01969781(98)00047-3

Dorman DC, Chiu W, Hales BF et al (2018) Polybrominated diphenyl ether (PBDE) neurotoxicity: a systematic review and metaanalysis of animal evidence. J Toxicol Environ Health B Crit Rev 21:269-289. https://doi.org/10.1080/10937404.2018.1514829

Drage DS, Heffernan AL, Cunningham TK et al (2019) Serum measures of hexabromocyclododecane (HBCDD) and polybrominated diphenyl ethers (PBDEs) in reproductive-aged women in the United Kingdom. Environ Res 177:108631. https://doi.org/10. 1016/j.envres.2019.108631

Dumais KM, Alonso AG, Immormino MA et al (2016) Involvement of the oxytocin system in the bed nucleus of the stria terminalis in the sex-specific regulation of social recognition. Psychoneuroendocrinology 64:79-88. https://doi.org/10.1016/j.psyneuen. 2015.11.007
Eskenazi B, Chevrier J, Rauch SA (2013) In Utero and Childhood Polybrominated Diphenyl Ether (PBDE) Exposures and Neurodevelopment in the CHAMACOS Study. Environmentalist

Ewbank MP, Pell PJ, Powell TE et al (2017) Repetition suppression and memory for faces is reduced in adults with autism spectrum conditions. Cereb Cortex 27:92-103. https://doi.org/10.1093/ cercor/bhw373

Fairless AH, Shah RY, Guthrie AJ et al (2011) Deconstructing sociability, an autism-relevant phenotype, in mouse models. Anat Rec 294:1713-1725. https://doi.org/10.1002/ar.21318

Ferguson JN, Young LJ, Hearn EF et al (2000) Social amnesia in mice lacking the oxytocin gene. Nat Genet 25:284-288. https://doi. org/10.1038/77040

Ferguson JN, Aldag JM, Insel TR, Young LJ (2001) Oxytocin in the medial amygdala is essential for social recognition in the mouse. J Neurosci 21:8278-8285

Fernie KJ, Shutt JL, Mayne G et al (2005) Exposure to polybrominated diphenyl ethers (PBDEs): changes in thyroid, vitamin A, glutathione homeostasis, and oxidative stress in American kestrels (Falco sparverius). Toxicol Sci 88:375-383

Ferretti V, Maltese F, Contarini G et al (2019) Oxytocin signaling in the central amygdala modulates emotion discrimination in mice. Curr Biol 29:1938-1953.e6. https://doi.org/10.1016/j.cub.2019. 04.070

Fischer D, Hooper K, Athanasiadou M et al (2006) Children show highest levels of polybrominated diphenyl ethers in a California family of four: a case study. Environ Health Perspect 114:1581-1584. https://doi.org/10.1289/ehp.8554

Frank E, Landgraf R (2008) The vasopressin system-from antidiuresis to psychopathology. Eur J Pharmacol 583:226-242. https:// doi.org/10.1016/j.ejphar.2007.11.063

Friard O, Gamba M (2016) BORIS: a free, versatile open-source event-logging software for video/audio coding and live observations. Methods Ecol Evol 7:1325-1330. https://doi.org/10. 1111/2041-210x.12584

Gascon M, Vrijheid M, Martínez D et al (2011) Effects of pre and postnatal exposure to low levels of polybromodiphenyl ethers on neurodevelopment and thyroid hormone levels at 4 years of age. Environ Int 37:605-611. https://doi.org/10.1016/j.envint. 2010.12.005

Gibson EA, Siegel EL, Eniola F et al (2018) Effects of polybrominated diphenyl ethers on child cognitive, behavioral, and motor development. Int J Environ Res Public Health. https://doi.org/ 10.3390/ijerph15081636

Gillera SEA, Marinello WP, Horman BM et al (2020) Sex-specific effects of perinatal FireMaster® 550 (FM 550) exposure on socioemotional behavior in prairie voles. Neurotoxicol Teratol 79:106840. https://doi.org/10.1016/j.ntt.2019.106840

Gillera SEA, Marinello WP, Cao KT et al (2021) Sex-specific disruption of the prairie vole hypothalamus by developmental exposure to a flame retardant mixture. Endocrinology. https://doi. org/10.1210/endocr/bqab100

Grandjean P, Landrigan PJ (2014) Neurobehavioural effects of developmental toxicity. Lancet Neurol 13:330-338. https://doi.org/ 10.1016/S1474-4422(13)70278-3

Guo W, Holden A, Smith SC et al (2016) PBDE levels in breast milk are decreasing in California. Chemosphere 150:505-513. https://doi.org/10.1016/j.chemosphere.2015.11.032

Hall CS (1934) Emotional behavior in the rat. I. Defecation and urination as measures of individual differences in emotionality. $\mathrm{J}$ Comp Psychol 18:385-403. https://doi.org/10.1037/h0071444

Hayashi R, Kasahara Y, Hidema S et al (2020) Oxytocin ameliorates impaired behaviors of high fat diet-induced obese mice. Front Endocrinol 11:379. https://doi.org/10.3389/fendo.2020.00379 
Hendaus MA, Jomha FA, Alhammadi AH (2019) Vasopressin in the amelioration of social functioning in autism spectrum disorder. J Clin Med Res. https://doi.org/10.3390/jcm8071061

Herbstman JB, Sjödin A, Kurzon M et al (2010) Prenatal exposure to PBDEs and neurodevelopment. Environ Health Perspect 118:712-719. https://doi.org/10.1289/ehp.0901340

Hernandez Scudder ME, Weinberg A, Thompson L et al (2020) Prenatal EDCs impair mate and odor preference and activation of the VMN in male and female rats. Endocrinology. https://doi. org/10.1210/endocr/bqaa124

Hess SE, Rohr S, Dufour BD et al (2008) Home improvement: C57BL/6J mice given more naturalistic nesting materials build better nests. J Am Assoc Lab Anim Sci 47:25-31

Hoffman K, Adgent M, Goldman BD et al (2012) Lactational exposure to polybrominated diphenyl ethers and its relation to social and emotional development among toddlers. Environ Health Perspect 120:1438-1442. https://doi.org/10.1289/ehp.1205100

Huckins LM, Logan DW, Sánchez-Andrade G (2013) Olfaction and olfactory-mediated behaviour in psychiatric disease models. Cell Tissue Res 354:69-80. https://doi.org/10.1007/ s00441-013-1617-7

Hurley S, Goldberg D, Nelson DO et al (2017) Temporal Evaluation of Polybrominated Diphenyl Ether (PBDE) Serum Levels in Middle-Aged and Older California Women, 2011-2015. Environ Sci Technol 51:4697-4704. https://doi.org/10.1021/ acs.est.7b00565

Ionas AC, Ulevicus J, Gómez AB et al (2016) Children's exposure to polybrominated diphenyl ethers (PBDEs) through mouthing toys. Environ Int 87:101-107. https://doi.org/10.1016/j.envint. 2015.11.018

Jiménez JA, Zylka MJ (2021) Controlling litter effects to enhance rigor and reproducibility with rodent models of neurodevelopmental disorders. J Neurodev Disord 13:2. https://doi.org/10. 1186/s11689-020-09353-y

Johnson-Restrepo B, Kannan K (2009) An assessment of sources and pathways of human exposure to polybrominated diphenyl ethers in the United States. Chemosphere 76:542-548. https:// doi.org/10.1016/j.chemosphere.2009.02.068

Kalueff AV, Keisala T, Minasyan A et al (2008) The regular and light-dark Suok tests of anxiety and sensorimotor integration: utility for behavioral characterization in laboratory rodents. Nat Protoc 3:129-136. https://doi.org/10.1038/nprot.2007.516

Kim YS, Leventhal BL, Koh Y-J et al (2011) Prevalence of autism spectrum disorders in a total population sample. Am J Psychiatry 168:904-912. https://doi.org/10.1176/appi.ajp.2011. 10101532

Kim B, Colon E, Chawla S et al (2015) Endocrine disruptors alter social behaviors and indirectly influence social hierarchies via changes in body weight. Environ Health 14:64. https://doi.org/ 10.1186/s12940-015-0051-6

Knobloch HS, Charlet A, Hoffmann LC et al (2012) Evoked axonal oxytocin release in the central amygdala attenuates fear response. Neuron 73:553-566. https://doi.org/10.1016/j.neuron.2011.11. 030

Kobayakawa K, Kobayakawa R, Matsumoto H et al (2007) Innate versus learned odour processing in the mouse olfactory bulb. Nature 450:503-508. https://doi.org/10.1038/nature06281

Kobylinska L, Panaitescu AM, Gabreanu G et al (2019) Plasmatic levels of neuropeptides, including oxytocin, in children with autism spectrum disorder, correlate with the disorder severity. Acta Endocrinol 5:16-24. https://doi.org/10.4183/aeb.2019.16

Kodavanti PRS, Curras-Collazo MC (2010) Neuroendocrine actions of organohalogens: thyroid hormones, arginine vasopressin, and neuroplasticity. Front Neuroendocrinol 31:479-496. https://doi. org/10.1016/j.yfrne.2010.06.005
Kodavanti PRS, Coburn CG, Moser VC et al (2010) Developmental exposure to a commercial PBDE mixture, DE-71: neurobehavioral, hormonal, and reproductive effects. Toxicol Sci 116:297312. https://doi.org/10.1093/toxsci/kfq105

Koenig CM, Lango J, Pessah IN, Berman RF (2012) Maternal transfer of BDE-47 to offspring and neurobehavioral development in C57BL/6J mice. Neurotoxicol Teratol 34:571-580. https://doi. org/10.1016/j.ntt.2012.09.005

Kogan JH, Franklandand PW, Silva AJ (2000) Long-term memory underlying hippocampus-dependent social recognition in mice. Hippocampus 10:47-56

Kozlova EV, Chinthirla BD, Pérez PA et al (2020) Maternal transfer of environmentally relevant polybrominated diphenyl ethers (PBDEs) produces a diabetic phenotype and disrupts glucoregulatory hormones and hepatic endocannabinoids in adult mouse female offspring. Sci Rep 10:18102. https://doi.org/10.1038/ s41598-020-74853-9

Kozlova EV, Carrillo V, Stapleton H, Curras-Collazo MC (2019) Neurotoxic effects of developmental exposure to DE-71 on forebrain social peptides, social behavior and olfaction in c57bl/6 mice. http://dioxin20xx.org/wp-content/uploads/pdfs/2019/1057.pdf. Accessed 3 May 2021

LaA Guardia MJ, Hale RC, Harvey E (2006) Detailed polybrominated diphenyl ether (PBDE) congener composition of the widely used penta-, octa-, and deca-PBDE technical flame-retardant mixtures. Environ Sci Technol 40:6247-6254. https://doi.org/10.1021/ es060630m

Lam J, Lanphear BP, Bellinger D et al (2017) Developmental PBDE Exposure and IQ/ADHD in Childhood: A Systematic Review and Meta-analysis. Environ Health Perspect 125:086001. https://doi. org/10.1289/EHP1632

Landgraf R, Neumann ID (2004) Vasopressin and oxytocin release within the brain: a dynamic concept of multiple and variable modes of neuropeptide communication. Front Neuroendocrinol 25:150-176. https://doi.org/10.1016/j.yfrne.2004.05.001

Legiša J, Messinger DS, Kermol E, Marlier L (2013) Emotional responses to odors in children with high-functioning autism: autonomic arousal, facial behavior and self-report. J Autism Dev Disord 43:869-879. https://doi.org/10.1007/s10803-012-1629-2

Li Z-M, Benker B, Bao Q et al (2020) Placental distribution of endogenous and exogenous substances: A pilot study utilizing cryo-sampled specimen off delivery room. Placenta 100:45-53. https://doi. org/10.1016/j.placenta.2020.08.009

Li Z, You M, Che X et al (2021) Perinatal exposure to BDE-47 exacerbated autistic-like behaviors and impairments of dendritic development in a valproic acid-induced rat model of autism. Ecotoxicol Environ Saf 212:112000. https://doi.org/10.1016/j. ecoenv.2021.112000

Lind Y, Darnerud PO, Atuma S et al (2003) Polybrominated diphenyl ethers in breast milk from Uppsala County, Sweden. Environ Res 93:186-194. https://doi.org/10.1016/s0013-9351(03)00049-5

Lipscomb ST, McClelland MM, MacDonald M et al (2017) Crosssectional study of social behaviors in preschool children and exposure to flame retardants. Environ Health 16:23. https://doi. org/10.1186/s12940-017-0224-6

Ludwig M, Apps D, Menzies J et al (2016) Dendritic release of neurotransmitters. Compr Physiol 7:235-252. https://doi.org/10.1002/ cphy.c160007

Lukas M, Bredewold R, Landgraf R et al (2011) Early life stress impairs social recognition due to a blunted response of vasopressin release within the septum of adult male rats. Psychoneuroendocrinology 36:843-853. https://doi.org/10.1016/j.psyne uen.2010.11.007

Luo M, Fee MS, Katz LC (2003) Encoding pheromonal signals in the accessory olfactory bulb of behaving mice. Science 299:11961201. https://doi.org/10.1126/science. 1082133 
Lyche JL, Rosseland C, Berge G, Polder A (2015) Human health risk associated with brominated flame-retardants (BFRs). Environ Int 74:170-180. https://doi.org/10.1016/j.envint.2014.09.006

Maenner MJ, Shaw KA, Baio J, et al (2020) Prevalence of autism spectrum disorder among children aged 8 Years - autism and developmental disabilities monitoring network, 11 Sites, United States, 2016. MMWR Surveill Summ 69:1-12. https://doi.org/ 10.15585/mmwr.ss6904a1

Mamrut S, Harony H, Sood R et al (2013) DNA methylation of specific $\mathrm{CpG}$ sites in the promoter region regulates the transcription of the mouse oxytocin receptor. PLoS ONE 8:e56869. https://doi. org/10.1371/journal.pone.0056869

Martin GN, Daniel N (2014) Autism spectrum disorders and chemoreception: dead-end or fruitful avenue of inquiry? Front Psychol 5:42. https://doi.org/10.3389/fpsyg.2014.00042

Messer A (2010) Mini-review: polybrominated diphenyl ether (PBDE) flame retardants as potential autism risk factors. Physiol Behav 100:245-249. https://doi.org/10.1016/j.physbeh.2010.01.011

Meyer-Lindenberg A, Domes G, Kirsch P, Heinrichs M (2011) Oxytocin and vasopressin in the human brain: social neuropeptides for translational medicine. Nat Rev Neurosci 12:524-538. https:// doi.org/10.1038/nrn3044

Mitchell MM, Woods R, Chi L-H et al (2012) Levels of select PCB and PBDE congeners in human postmortem brain reveal possible environmental involvement in 15q11-q13 duplication autism spectrum disorder. Environ Mol Mutagen 53:589-598. https:// doi.org/10.1002/em.21722

Morais LH, Golubeva AV, Moloney GM et al (2020) Enduring behavioral effects induced by birth by caesarean section in the mouse. Curr Biol 30:3761-3774.e6. https://doi.org/10.1016/j.cub.2020. 07.044

Moy SS, Nadler JJ, Perez A et al (2004) Sociability and preference for social novelty in five inbred strains: an approach to assess autistic-like behavior in mice. Genes Brain Behav 3:287-302. https://doi.org/10.1111/j.1601-1848.2004.00076.x

Mucio-Ramírez S, Sánchez-Islas E, Sánchez-Jaramillo E et al (2017) Perinatal exposure to organohalogen pollutants decreases vasopressin content and its mRNA expression in magnocellular neuroendocrine cells activated by osmotic stress in adult rats. Toxicol Appl Pharmacol 329:173-189. https://doi.org/10.1016/j. taap.2017.05.039

Murai T, Okuda S, Tanaka T, Ohta H (2007) Characteristics of object location memory in mice: Behavioral and pharmacological studies. Physiol Behav 90:116-124. https://doi.org/10.1016/j.physb eh.2006.09.013

Nagarajan RP, Hogart AR, Gwye Y et al (2006) Reduced MeCP2 expression is frequent in autism frontal cortex and correlates with aberrant MECP2 promoter methylation. Epigenetics 1:e111. https://doi.org/10.4161/epi.1.4.3514

Noack J, Richter K, Laube G et al (2010) Different importance of the volatile and non-volatile fractions of an olfactory signature for individual social recognition in rats versus mice and short-term versus long-term memory. Neurobiol Learn Mem 94:568-575. https://doi.org/10.1016/j.nlm.2010.09.013

Oettl L-L, Ravi N, Schneider M et al (2016) Oxytocin enhances social recognition by modulating cortical control of early olfactory processing. Neuron 90:609-621. https://doi.org/10.1016/j.neuron. 2016.03.033

Ohajinwa CM, Van Bodegom PM, Xie Q et al (2019) Hydrophobic organic pollutants in soils and dusts at electronic waste recycling sites: occurrence and possible impacts of polybrominated diphenyl ethers. Int J Environ Res Public Health. https://doi.org/ 10.3390/ijerph 16030360

Oztan O, Garner JP, Partap S et al (2018) Cerebrospinal fluid vasopressin and symptom severity in children with autism. Ann Neurol 84:611-615. https://doi.org/10.1002/ana.25314
Palkovits M (1973) Isolated removal of hypothalamic or other brain nuclei of the rat. Brain Res 59:449-450. https://doi.org/10.1016/ 0006-8993(73)90290-4

Patisaul HB (2017) Endocrine disruption of vasopressin systems and related behaviors. Front Endocrinol 8:134. https://doi.org/10. 3389/fendo.2017.00134

Pelch KE, Bolden AL, Kwiatkowski CF (2019) Environmental chemicals and autism: a scoping review of the human and animal research. Environ Health Perspect 127:46001. https://doi.org/ 10.1289/EHP4386

Pfaffl MW (2001) A new mathematical model for relative quantification in real-time RT-PCR. Nucleic Acids Res 29:e45-e45. https://doi. org/10.1093/nar/29.9.e45

Pinson A, Bourguignon JP, Parent AS (2016) Exposure to endocrine disrupting chemicals and neurodevelopmental alterations. Andrology 4:706-722. https://doi.org/10.1111/andr.12211

Poston RG, Saha RN (2019) Epigenetic Effects of Polybrominated Diphenyl Ethers on Human Health. Int J Environ Res Public Health. https://doi.org/10.3390/ijerph16152703

Raam T, McAvoy KM, Besnard A et al (2017) Hippocampal oxytocin receptors are necessary for discrimination of social stimuli. Nat Commun 8:2001. https://doi.org/10.1038/s41467-017-02173-0

Reilly MP, Weeks CD, Topper VY et al (2015) The effects of prenatal PCBs on adult social behavior in rats. Horm Behav 73:47-55. https://doi.org/10.1016/j.yhbeh.2015.06.002

Reilly MP, Kunkel MN, Thompson LM et al (2021) Effects of endocrine-disrupting chemicals on hypothalamic oxytocin and vasopressin systems. J Exp Zool A Ecol Integr Physiol. https://doi. org/10.1002/jez.2475

Rogers SJ, Hepburn S, Wehner E (2003) Parent reports of sensory symptoms in toddlers with autism and those with other developmental disorders. J Autism Dev Disord 33:631-642. https://doi. org/10.1023/b:jadd.0000006000.38991.a7

Rose M, Bennett DH, Bergman A et al (2010) PBDEs in 2-5 year-old children from California and associations with diet and indoor environment. Environ Sci Technol 44:2648-2653. https://doi.org/ 10.1021/es $903240 \mathrm{~g}$

Roze E, Meijer L, Bakker A et al (2009) Prenatal exposure to organohalogens, including brominated flame retardants, influences motor, cognitive, and behavioral performance at school age. Environ Health Perspect 117:1953-1958. https://doi.org/10.1289/ehp. 0901015

Rynkiewicz A, Janas-Kozik M, Słopień A (2019) Girls and women with autism. Psychiatr Pol 53:737-752. https://doi.org/10.12740/ PP/OnlineFirst/95098

Sanders JM, Lebetkin EH, Chen L-J, Burka LT (2006) Disposition of 2,2',4,4',5,5'-hexabromodiphenyl ether (BDE153) and its interaction with other polybrominated diphenyl ethers (PBDEs) in rodents. Xenobiotica 36:824-837. https://doi.org/10.1080/00498 250600815906

Schecter A, Johnson-Welch S, Tung KC et al (2007) Polybrominated diphenyl ether (PBDE) levels in livers of U.S. human fetuses and newborns. J Toxicol Environ Health A 70:1-6. https://doi.org/10. 1080/15287390600748369

Schecter A, Päpke O, Tung KC et al (2005) Polybrominated Diphenyl Ether Flame Retardants in the U.S. Population: Current Levels, Temporal Trends, and Comparison With Dioxins, Dibenzofurans, and Polychlorinated Biphenyls. J Occup Environ Med 47:199. https://doi.org/10.1097/01.jom.0000158704.27536.d2

Sgritta M, Dooling SW, Buffington SA et al (2019) Mechanisms underlying microbial-mediated changes in social behavior in mouse models of autism spectrum disorder. Neuron 101:246-259.e6. https://doi.org/10.1016/j.neuron.2018.11.018

Silverman JL, Yang M, Lord C, Crawley JN (2010) Behavioural phenotyping assays for mouse models of autism. Nat Rev Neurosci 11:490-502. https://doi.org/10.1038/nrn2851 
Social processes: Workshop proceedings (2012). https://www.nimh. nih.gov/research/research-funded-by-nimh/rdoc/social-proce sses-workshop-proceedings. Accessed 6 Sep 2021

Stapleton HM, Dodder NG, Offenberg JH et al (2005) Polybrominated diphenyl ethers in house dust and clothes dryer lint. Environ Sci Technol 39:925-931. https://doi.org/10.1021/es0486824

Stapleton HM, Allen JG, Kelly SM et al (2008) Alternate and new brominated flame retardants detected in US house dust. Environ Sci Technol 42:6910-6916

Stapleton HM, Eagle S, Sjödin A, Webster TF (2012) Serum PBDEs in a North Carolina toddler cohort: associations with handwipes, house dust, and socioeconomic variables. Environ Health Perspect 120:1049-1054. https://doi.org/10.1289/ehp.1104802

Staskal DF, Hakk H, Bauer D et al (2006) Toxicokinetics of polybrominated diphenyl ether congeners 47,99,100, and 153 in mice. Toxicol Sci 94:28-37. https://doi.org/10.1093/toxsci/kfl091

Suvorov A, Vandenberg LN (2016) To cull or not to cull? considerations for studies of endocrine-disrupting chemicals. Endocrinology 157:2586-2594. https://doi.org/10.1210/en.2016-1145

Ta TA, Koenig CM, Golub MS et al (2011) Bioaccumulation and behavioral effects of 2,2',4,4'-tetrabromodiphenyl ether (BDE47) in perinatally exposed mice. Neurotoxicol Teratol 33:393404. https://doi.org/10.1016/j.ntt.2011.02.003

Takayanagi Y, Yoshida M, Takashima A et al (2017) Activation of supraoptic oxytocin neurons by secretin facilitates social recognition. Biol Psychiatry 81:243-251. https://doi.org/10.1016/j.biops ych.2015.11.021

Tanimizu T, Kenney JW, Okano E et al (2017) Functional connectivity of multiple brain regions required for the consolidation of social recognition memory. J Neurosci 37:4103-4116. https://doi.org/ 10.1523/JNEUROSCI.3451-16.2017

Terasaki LS, Gomez J, Schwarz JM (2016) An examination of sex differences in the effects of early-life opiate and alcohol exposure. Philos Trans R Soc Lond B Biol Sci 371:20150123. https://doi. org/10.1098/rstb.2015.0123

Terry P, Towers CV, Liu L-Y et al (2017) Polybrominated diphenyl ethers (flame retardants) in mother-infant pairs in the Southeastern U.S. Int J Environ Health Res 27:205-214. https://doi.org/ 10.1080/09603123.2017.1332344

Thomas A, Burant A, Bui N et al (2009) Marble burying reflects a repetitive and perseverative behavior more than novelty-induced anxiety. Psychopharmacology 204:361-373. https://doi.org/10. 1007/s00213-009-1466-y

Toms L-ML, Harden F, Paepke O et al (2008) Higher accumulation of polybrominated diphenyl ethers in infants than in adults. Environ Sci Technol 42:7510-7515. https://doi.org/10.1021/es800719v

Topper VY, Reilly MP, Wagner LM et al (2019) Social and neuromolecular phenotypes are programmed by prenatal exposures to endocrine-disrupting chemicals. Mol Cell Endocrinol 479:133146. https://doi.org/10.1016/j.mce.2018.09.010

Veenema AH, Bredewold R, De Vries GJ (2012) Vasopressin regulates social recognition in juvenile and adult rats of both sexes, but in sex- and age-specific ways. Horm Behav 61:50-56. https://doi. org/10.1016/j.yhbeh.2011.10.002

Viberg H, Fredriksson A, Eriksson P (2003) Neonatal exposure to polybrominated diphenyl ether (PBDE 153) disrupts spontaneous behaviour, impairs learning and memory, and decreases hippocampal cholinergic receptors in adult mice. Toxicol Appl Pharmacol 192:95-106. https://doi.org/10.1016/s0041-008x(03) 00217-5

Viberg H, Fredriksson A, Eriksson P (2004) Investigations of strain and/or gender differences in developmental neurotoxic effects of polybrominated diphenyl ethers in mice. Toxicol Sci 81:344-353. https://doi.org/10.1093/toxsci/kfh215

Vuong AM, Yolton K, Xie C et al (2017) Childhood polybrominated diphenyl ether (PBDE) exposure and neurobehavior in children at 8 years. Environ Res 158:677-684. https://doi.org/10.1016/j. envres.2017.07.028

Vuong AM, Yolton K, Dietrich KN et al (2018) Exposure to polybrominated diphenyl ethers (PBDEs) and child behavior: Current findings and future directions. Horm Behav 101:94-104. https:// doi.org/10.1016/j.yhbeh.2017.11.008

Wacker DW, Ludwig M (2012) Vasopressin, oxytocin, and social odor recognition. Horm Behav 61:259-265. https://doi.org/10.1016/j. yhbeh.2011.08.014

Wang D, Yan J, Teng M et al (2018) In utero and lactational exposure to BDE-47 promotes obesity development in mouse offspring fed a high-fat diet: impaired lipid metabolism and intestinal dysbiosis. Arch Toxicol 92:1847-1860. https://doi.org/10.1007/ s00204-018-2177-0

Weigelt S, Koldewyn K, Kanwisher N (2012) Face identity recognition in autism spectrum disorders: a review of behavioral studies. Neurosci Biobehav Rev 36:1060-1084. https://doi.org/10.1016/j. neubiorev.2011.12.008

Werling DM, Geschwind DH (2013) Sex differences in autism spectrum disorders. Curr Opin Neurol 26:146-153. https://doi.org/ 10.1097/WCO.0b013e32835ee548

Witchey SK, Al Samara L, Horman BM et al (2020) Perinatal exposure to FireMaster ${ }^{\circledR} 550$ (FM550), brominated or organophosphate flame retardants produces sex and compound specific effects on adult Wistar rat socioemotional behavior. Horm Behav 126:104853. https://doi.org/10.1016/j.yhbeh.2020.104853

Woods R, Vallero RO, Golub MS et al (2012) Long-lived epigenetic interactions between perinatal PBDE exposure and Mecp2308 mutation. Hum Mol Genet 21:2399-2411. https://doi.org/10. 1093/hmg/dds046

Xu M, Huang Y, Li K et al (2018) Developmental exposure of decabromodiphenyl ether impairs subventricular zone neurogenesis and morphology of granule cells in mouse olfactory bulb. Arch Toxicol 92:529-539. https://doi.org/10.1007/s00204-017-2059-x

Yang M, Silverman JL, Crawley JN (2011) Automated three-chambered social approach task for mice. Curr Protoc Neurosci. https://doi. org/10.1002/0471142301.ns0826s56

Young LJ, Pitkow LJ, Ferguson JN (2002) Neuropeptides and social behavior: animal models relevant to autism. Mol Psychiatry 7(Suppl 2):S38-S39. https://doi.org/10.1038/sj.mp.4001175

Zhang H-F, Dai Y-C, Wu J et al (2016) Plasma oxytocin and argininevasopressin levels in children with autism spectrum disorder in china: associations with symptoms. Neurosci Bull 32:423-432. https://doi.org/10.1007/s12264-016-0046-5

Zhang Y, Li N, Li C et al (2020) Genetic evidence of gender difference in autism spectrum disorder supports the female-protective effect. Transl Psychiatry 10:4. https://doi.org/10.1038/ s41398-020-0699-8

Zhao Y, Ruan X, Li Y et al (2013) Polybrominated diphenyl ethers (PBDEs) in aborted human fetuses and placental transfer during the first trimester of pregnancy. Environ Sci Technol 47:59395946. https://doi.org/10.1021/es305349x

Publisher's Note Springer Nature remains neutral with regard to jurisdictional claims in published maps and institutional affiliations. 


\section{Authors and Affiliations}

Elena V. Kozlova ${ }^{1,2}$ - Matthew C. Valdez ${ }^{1,2,8} \cdot$ Maximillian E. Denys $^{1} \cdot$ Anthony E. Bishay $^{1}{ }^{\mathbb{D}} \cdot$ Julia M. Krum $^{1}$. Kayhon M. Rabbani ${ }^{1}$. Valeria Carrillo ${ }^{1}$. Gwendolyn M. Gonzalez ${ }^{1}$. Gregory Lampel ${ }^{1}$. Jasmin D. Tran ${ }^{1}$. Brigitte M. Vazquez ${ }^{1}$. Laura M. Anchondo ${ }^{1}$ - Syed A. Uddin ${ }^{1}$ - Nicole M. Huffman ${ }^{1}$ - Eduardo Monarrez ${ }^{1}$. Duraan S. Olomi ${ }^{1}$ - Bhuvaneswari D. Chinthirla ${ }^{1} \cdot$ Richard E. Hartman $^{4} \cdot$ Prasada Rao S. Kodavanti $^{8}$. Gladys Chompre $^{5}$ - Allison L. Phillips ${ }^{3} \cdot$ Heather M. Stapleton $^{3} \cdot$ Bernhard Henkelmann $^{6} \cdot$ Karl-Werner Schramm $^{6,7}\left({ }^{\circ}\right.$. Margarita C. Curras-Collazo ${ }^{1}$ [D

1 Department of Molecular, Cell and Systems Biology, University of California, Riverside, CA 92521, USA

2 Neuroscience Graduate Program, University of California, Riverside, CA 92521, USA

3 Duke University, Nicholas School of the Environment, Durham, NC 27710, USA

4 Department of Psychology, Loma Linda University, Loma Linda, CA 92350, USA

5 Biotechnology Department, Pontifical Catholic University of Puerto Rico, Ponce, Puerto Rico 00717-9997, USA

6 Helmholtz Zentrum Munchen, Molecular EXposomics (MEX), German National Research Center for Environmental
Health $(\mathrm{GmbH})$, Ingolstaedter Landstrasse 1, Neuherberg, Munich, Germany

7 Department Für Biowissenschaftliche Grundlagen, TUM, Wissenschaftszentrum Weihenstephan für Ernährung, Landnutzung Und Umwelt, Weihenstephaner Steig 23, 85350 Freising, Germany

8 Neurological and Endocrine Toxicology Branch, Public Health and Integrated Toxicology Division, CPHEA/ORD, U.S. Environmental Protection Agency, Research Triangle Park, Durham, NC 27711, USA 